\title{
The effect of extra dimensions on gravity wave bursts from cosmic string cusps
}

\author{
Eimear O’Callaghan ${ }^{a *}$, Sarah Chadburn ${ }^{b \dagger}$, Ghazal Geshnizjani $^{c}$, \\ Ruth Gregory ${ }^{a, b}$, Ivonne Zavala ${ }^{d}$ ฯ \\ ${ }^{a}$ Institute for Particle Physics Phenomenology, Department of Physics, \\ Durham University, South Road, Durham, DH1 3LE, UK \\ ${ }^{b}$ Centre for Particle Theory, Department of Mathematical Sciences, Durham \\ University, South Road, Durham, DH1 3LE, UK \\ c Perimeter Institute for Theoretical Physics, 31 Caroline Street North, \\ Waterloo ON, N2L 2Y, Canada. \\ ${ }^{d}$ Bethe Center for Theoretical Physics and Physikalisches Institut der \\ Universität Bonn, Nußallee 12, D-53115 Bonn, Germany.
}

\begin{abstract}
We explore the kinematical effect of having extra dimensions on the gravitational wave emission from cosmic strings. Additional dimensions both round off cusps, and reduce the probability of their formation. We recompute the gravitational wave burst, taking into account these two factors, and find a potentially significant damping on the gravitational waves of the strings.
\end{abstract}

KEywords: Large Extra Dimensions, Cosmic Superstrings.

\footnotetext{
*Email: e.e.o'callaghan@durham.ac.uk

${ }^{\dagger}$ Email: s.e.chadburn@durham.ac.uk

${ }^{\ddagger}$ Email: ggeshnizjani@perimeterinstitute.ca

§Email: r.a.w.gregory@durham.ac.uk

๑Email: zavala@th.physik.uni-bonn.de
} 


\section{Contents}

1. Introduction 1

2. String motion and cusps 4

3. Gravitational waves from cusps 6

4. Wave form in extra dimensions 9

4.1 String kinematics with extra dimensions 10

4.2 The gravitational waveform 13

5. Discussion 18

A. Finite width corrections to the cusp 22

\section{Introduction}

The notion that nature might have extra dimensions, and that these might leave traces in observation or experiment, has led to many interesting ideas and advances over the last decade. Traditionally, extra dimensions would be hidden via a KaluzaKlein mechanism, accessible only at ultra high energies. More recently however, the braneworld paradigm, [1, 2, 3], has allowed for extra dimensions to be much larger, hidden instead by a confinement mechanism, [1], which localizes standard model physics on the brane but allows gravity to probe the bulk. These Large Extra Dimension (LED) scenarios, [2, 3], have the added attraction of providing a natural hierarchy between gauge and gravity interactions coming from geometric multiplying factors in the derived 4-dimensional Planck mass. These ideas have been incorporated into string theory models, allowing LED's via a process of flux stabilization, [⿴囗十.

One of the advantages of the braneworld scenarios was that they gave concrete predictions for cosmology. In particular, the Randall-Sundrum (RS), [3] set-up, with a brane living in anti de Sitter (adS) spacetime with one extra dimension, has a simple cosmology, the scale factor being determined by motion through a black hole spacetime, [5]. This led to the mirage picture, [6], in which brane cosmology is determined by bulk motion through a warped space, however, the mirage picture does not explicitly include the gravitational back reaction of the braneworld, which for codimension two and higher can be problematic, [7]. 
Nonetheless, the idea of the braneworld - an object localized in extra dimensions - has proven to be extremely useful when constructing models of inflation in string theory. The localization in extra dimensions provides a natural scalar field (the position of the brane) which can then evolve according to some effective action depending on the specifics of the internal manifold and the brane itself. Early models of brane inflation, [8], used the Newtonian potential of the higher dimensional solution to provide the inflaton potential. This however, was not consistent with the size of the extra dimensions, nor was any mechanism offered for their stabilization. However, with the completion of the picture via flux stabilization, [因, 9], a great many models of brane inflation have been explored (see [10] for a review).

A key side effect of brane inflation is the formation of cosmic strings, [11, 12]. These strings form as a by product of the annihilation of the inflationary branes (for reviews see [13]), and while having their origins in superstring theory, can have a wide range of parameters, and interesting physical properties.

Cosmic strings, [14, are an example of a topological defect, a glitch in the vacuum structure of a field theory which can arise when the vacuum manifold is topologically nontrivial. They are ubiquitous in all types of physics, from condensed matter systems to quantum field theory. They were first explored in the cosmological setting by Zeldovich and Kibble, [15], who realised that they could arise in the early universe as possible side-products of symmetry breaking phase transitions. Indeed, it was the cosmological catastrophe of monopole production at the GUT scale that in part led to the development of the original inflationary scenario. Cosmic strings however are cosmologically benign, and exhibit a scaling network behaviour, [16]. Accounting for their gravitational effects made them a possible candidate for the perturbation spectrum until CMB experiments ruled them out, 17]. Nonetheless, the possibility remains that a network of light strings could be present in our universe, [18].

From the cosmological point of view, the internal structure of the cosmic string is irrelevant, and what we need is the long range behaviour of the string. Gravitationally, a straight string produces a conical deficit in spacetime [19], which, while it does lead to interesting lensing effects, cannot be regarded as a detection tool as it requires the serendipity of an appropriately aligned source behind the string (although see [20] for a discussion of detection via weak lensing). Instead, a more promising approach is to take the dynamical network of strings, and to use linearized gravity to compute the radiation emitted from the loops and crinkly long strings [21, 22, 23]. Network simulations approximate the string by the Nambu action:

$$
S=-\mu \int d^{2} \sigma \sqrt{\gamma}
$$

which can be rigorously derived from an underlying field theory model [24]. Together with rules for intercommutation [25], or how strings behave when they cross each 
other, this gives the basic physics of how a network evolves.

The primary drivers of network evolution are therefore whether strings intersect, and whether the motion has any extreme events (i.e. are there points at which the Nambu approximation is likely to break down). Early work focussed on taking toy families of loops, [26, 27], to explore the likelihood of self intersection, and also events where the string bends on the same scale as its width. The kinematics of strings obeying the Nambu action are very well understood: the picture is that loops often self intersect, and generically have cusps - points at which the string reaches the speed of light and its extrinsic curvature diverges, leading to a breakdown of the Nambu action. Finite width corrections to the Nambu action can be computed, [28, 29], but it was believed that these would not be significant for the network evolution. In appendix $\mathrm{A}$ we demonstrate this explicitly by computing the finite width effect for a cusp. More recently, further analysis of the effect of small scale structure on the string, [30], has indicated that there may actually be measurable consequences. In either case however, the primary importance of a cusp is that it acts as a strong source of gravitational radiation.

In a seminal paper, Damour and Vilenkin (DV), [31], re-examined gravitational radiation from cosmic strings, assessing for what range of mass per unit length the string could potentially be visible to the next range of gravitational wave detectors. The main effect they were considering was the burst of radiation from extreme kinematic events in the loop motion, known as cusps and kinks. They computed the amplitude of a cusp and kink gravitational wave burst (GWB) as a function of the mass per unit length of the string. In a later paper, [32], they allowed for networks formed by strings with lower intercommutation probabilities, which enhance the density and thus the GWB amplitude. Siemens et al. [33, 34, performed a more careful analysis of the cosmological expansion history, instead computing rates of events at amplitudes fixed by the detector. Since then, many other gravitational effects of cosmic superstrings have been explored, including strings with junctions, [35, and broken strings, 36.

In a recent note [37, we revisited the calculations of DV et al. arguing that the kinematic effect of the extra dimensions significantly reduced the power of the cusp waveform. Essentially, extra dimensions act to 'slow down' the string, as first pointed out by Avgoustidis and Shellard, [38], and round off the sharp cusp. In this paper we give a full computation of this effect, presenting test loop families to demonstrate parameter space measures, and detailed numerical calculations of GWB event rates and amplitudes.

The extra dimensions give two main modifications to the DV result. The first is the cusp rounding effect, and we show how this gives a high frequency cut-off to the gravitational waveform. The second is a probabilistic factor: in $3+1$ dimensions, cusps always form on a smooth loop trajectory, however, in higher dimensions this is no longer the case. DV introduced a parameter in their GWB calculation, $\mathcal{C}$, which 
measured the probability of cusp formation in a single loop period; the reason for introducing this in $3+1$ dimensions is that once strings intersect, they can have kinks, which lower the cusp probability. In higher dimensions however, the probability of cusp formation is strictly zero, in that the set of solutions which have cusps has measure zero. We therefore define a near cusp event, which represents the rounded cusp, and compute the probability of this event to input in the amplitude calculation.

Finally, one has to factor in the reduction in intercommutation probability due to the extra dimensions, [39, 40], which acts to increase the density of the network. Summing all these effects produces a marked effect on the GWB amplitude, and our conclusion is that detection of GWB's from cosmic superstrings by current or next generation gravitational wave detectors will be harder than suspected, with the bonus that positive detection may tell us something about the number of extra dimensions. Clearly, our result will also relax bounds on $G \mu$ for cosmic superstrings derived using the DV results 41 .

We start by reviewing the standard Kibble-Turok method of analysing string trajectories, [26], noting the new features that appear with additional dimensions. We then review the DV calculation of the GWB. Next we calculate the various effects coming from extra dimensions, introducing a parameter $\Delta$ which measures deviation from an exact cusp. We integrate over $\Delta$ to obtain the sum of all cusp or near cusp events, and finally discuss implications and caveats of the calculation. To be specific, we focus on the frequency band of the advanced LIGO detector, however we will comment on the frequency dependence of our results.

\section{String motion and cusps}

We begin by briefly reviewing the kinematics of cosmic strings, deriving the general form of a string solution and showing how cusps are generic. This formulation was largely developed by Kibble and Turok, [26], and is the standard method for finding loop trajectories.

Let $X^{\mu}\left(\sigma^{A}\right)$ be the spacetime coordinates of the string worldsheet, where $\sigma^{A}=$ $\{\tau, \sigma\}$ are intrinsic coordinates on the worldsheet. For closed loops, which we will be considering in this paper, $\sigma \in[0, L]$, where $L$ is the length of the loop. The induced metric on the worldsheet appearing in (1.1) is then:

$$
\gamma_{A B}=\frac{\partial X^{\mu}}{\partial \sigma^{A}} \frac{\partial X^{\nu}}{\partial \sigma^{B}} g_{\mu \nu}
$$

where $g_{\mu \nu}$ is the spacetime metric ${ }^{1}$. The Nambu-Goto action (1.1) is then proportional to the area of the string worldsheet, with the constant of proportionality being $\mu$, the tension, or mass per unit length of the string. Note that cosmic strings have a tension along their length equal to their energy density.

\footnotetext{
${ }^{1}$ We use a mostly minus signature.
} 
Because we are dealing with a two-dimensional metric, we can always choose a gauge in which $\gamma$ is conformally flat:

$$
\begin{array}{r}
\dot{X}^{\mu} X^{\nu} g_{\mu \nu}=0 \\
\left(\dot{X}^{\mu} \dot{X}^{\nu}+X^{\prime \mu} X^{\nu}\right) g_{\mu \nu}=0
\end{array}
$$

where a dot denotes $\partial / \partial \tau$ and a prime $\partial / \partial \sigma$. Kibble and Turok then chose the spacetime coordinates to coincide with the centre of mass frame of the string, and the worldsheet time coordinate to correspond with the spacetime time (temporal gauge). Thus writing $X^{\mu}=(\tau, \mathbf{r}(\tau, \sigma))$, we have:

$$
\begin{aligned}
\dot{\mathbf{r}} \cdot \mathbf{r}^{\prime} & =0 \\
\dot{\mathbf{r}}^{2}+\mathbf{r}^{\prime 2} & =1 \\
\ddot{\mathbf{r}}-\mathbf{r}^{\prime \prime} & =0
\end{aligned}
$$

where the first two correspond to the gauge constraints, and the final equation is the wave equation of motion for the string. It is conventional to use lightcone coordinates:

$$
\sigma_{ \pm}=\tau \pm \sigma
$$

in which the solutions to the equation of motion (2.6) take the form of left and right moving waves, conventionally written in the form

$$
\mathbf{r}=\frac{1}{2}\left[\mathbf{a}\left(\sigma_{-}\right)+\mathbf{b}\left(\sigma_{+}\right)\right]
$$

where the gauge conditions constrain $\mathbf{a}^{\prime}$ and $\mathbf{b}^{\prime}$ to lie on a unit sphere, commonly dubbed the "Kibble-Turok" sphere:

$$
\mathbf{a}^{\prime 2}=\mathbf{b}^{\prime 2}=1
$$

Notice that while the periodicity of $\mathbf{a}$ and $\mathbf{b}$ is $L$, the periodicity of the actual motion of the string is $L / 2$, since $\mathbf{r}(\sigma+L / 2, \tau+L / 2)=\mathbf{r}(\sigma, \tau)$.

There is an additional constraint that must be satisfied by both $\mathbf{a}^{\prime}$ and $\mathbf{b}^{\prime}$, for consistency with the facts that the loop is closed, and that we are in the c.o.m. frame. The former condition requires that $\mathbf{r}(\tau, 0)=\mathbf{r}(\tau, L)$, hence

$$
\int_{0}^{L} \mathbf{r}^{\prime} d \sigma=\int_{0}^{L}\left(\mathbf{b}^{\prime}-\mathbf{a}^{\prime}\right) d \sigma=0 .
$$

The latter condition requires the average momentum integrated along the string to vanish, i.e.:

$$
\int_{0}^{L} \dot{\mathbf{r}} d \sigma=\int_{0}^{L}\left(\mathbf{b}^{\prime}+\mathbf{a}^{\prime}\right) d \sigma=0
$$


thus

$$
\int_{0}^{L} \mathbf{b}^{\prime} d \sigma=\int_{0}^{L} \mathbf{a}^{\prime} d \sigma=0
$$

Hence $\mathbf{a}^{\prime}$ and $\mathbf{b}^{\prime}$ follow trajectories on a unit sphere with zero weight - their average position is the origin. Since they both define curves on a two dimensional manifold which must cover both halves of the sphere equally, they will in general cross. Inserting the expression for $\mathbf{r}$ into the intrinsic metric (2.1) gives:

$$
\gamma_{A B}=\frac{1}{2}\left(1-\mathbf{a}^{\prime} \cdot \mathbf{b}^{\prime}\right) \eta_{A B}
$$

thus when $\mathbf{a}^{\prime}$ and $\mathbf{b}^{\prime}$ are collinear, the metric becomes degenerate and a point of the worldsheet instantaneously reaches the speed of light. Strictly speaking the mass concentration on the string is infinite at this point, however as it has zero area the total energy is finite. However, since the vicinity of this point is highly relativistic, this rapidly moving part of the worldsheet will have high momentum, and hence we expect some significant gravitational interaction. Cusps are thus transient but powerful events; moreover, they are generic on string trajectories (notwithstanding the effect of small scale structure [30]). We now turn to a summary of the gravitational effects of cusps.

\section{Gravitational waves from cusps}

It is worth reviewing the Damour-Vilenkin argument, [31, as the derivation of the gravitational wave signal is quite involved and lengthy ${ }^{2}$. Damour and Vilenkin first computed the linearized metric perturbation arising from a single cusp event on a cosmic string loop of length $L$ in flat spacetime. The waveform of the cusp was found to have a power law behaviour of $f^{-4 / 3}$ (or $f^{-1 / 3}$ in their logarithmic Fourier representation) at large frequencies, $f$, of the gravitational wave. They then used this flat spacetime waveform to infer the cosmological waveform behaviour in the geometric optics limit, thus deriving a gravitational wave amplitude of a single cusp event which decays quite strongly with redshift. Finally, by considering a one scale model for the string network, they computed an event rate for observing cusp GWB's which increased rapidly with redshift $z$. By choosing a physically reasonable event rate, and picking a fiducial experimentally motivated frequency, they determined the typical redshift contributing to the GWB and calculated the amplitude of the cusp signal, presenting the results as a function of $G \mu$.

In order to present an analytic argument, DV introduced various interpolating functions in redshift space, and approximated at various stages the exact expressions in the waveform. As we review their argument, we will keep these exact expressions

\footnotetext{
${ }^{2}$ Note, DV use the mostly plus metric convention, hence some equations will have relative minus signs compared with those we present here.
} 
until the final stage of the calculation. When adding in the effect of the extra dimensions, we will first follow the same game as DV, introducing the same interpolating functions so that a direct comparison can be made. For interest however, we also include an exact numerical redshift integration.

The first step is to calculate the gravitational wave of a cosmic string loop in flat spacetime. We therefore need to solve the linearized Einstein equations

$$
\square \bar{h}_{\mu \nu}=-16 \pi G T_{\mu \nu}
$$

which in the far field approximation is given by

$$
\bar{h}_{\mu \nu} \simeq \frac{4 G}{r} \sum_{\omega} e^{-i \omega(t-r)} T_{\mu \nu}(\mathbf{k}, \omega)
$$

where $T_{\mu \nu}(\mathbf{k}, \omega)$ is the Fourier transformed energy momentum.

The energy momentum of the cosmic string is

$$
T^{\mu \nu}=\mu \int d^{2} \sigma\left(\dot{X}^{\mu} \dot{X}^{\nu}-X^{\prime \mu} X^{\prime \nu}\right) \delta^{(4)}\left(x^{\mu}-X^{\mu}(\sigma, \tau)\right)
$$

which means the gravitational wave is determined by the Fourier transform

$$
T^{\mu \nu}(\mathbf{k}, \omega)=\frac{\mu}{T_{L}} \int_{0}^{T_{L}} d \tau \int_{0}^{L} d \sigma \dot{X}_{+}^{(\mu} \dot{X}_{-}^{\nu)} e^{-\frac{i}{2}\left(k \cdot X_{+}+k \cdot X_{-}\right)}
$$

where $X_{+}^{\mu}=\left(\sigma_{+}, \mathbf{b}\left(\sigma_{+}\right)\right), X_{-}^{\mu}=\left(\sigma_{-}, \mathbf{a}\left(\sigma_{-}\right)\right)$, and a dot now denotes a derivative with respect to the argument of $X_{ \pm}^{\mu} ; k^{\mu}=\frac{4 \pi m}{L}(1, \mathbf{n})=m \omega_{L}(1, \mathbf{n})$ is the null wave vector. Here, $\omega_{L}$ is the frequency of the fundamental mode of the string loop.

A cusp corresponds to a lining up of the momenta of the left and right moving modes on the string loop: $\dot{X}_{+}^{\mu}=\dot{X}_{-}^{\mu}=\ell^{\mu}=\left(1, \mathbf{n}^{\prime}\right)$. Choosing the coordinate origins, we may write

$$
X_{ \pm}^{\mu}\left(\sigma_{ \pm}\right)=\ell^{\mu} \sigma_{ \pm}+\frac{1}{2} \ddot{X}_{0 \pm}^{\mu} \sigma_{ \pm}^{2}+\frac{1}{6} \dddot{X}_{0 \pm}^{\mu} \sigma_{ \pm}^{3}
$$

where the subscript 0 refers to evaluation at $\sigma_{ \pm}=0$. Now, defining the angle between $k^{\mu}$ and $\ell^{\mu}$ as $\theta$, which is assumed to be small, and writing $d^{\mu}=k^{\mu}-\ell^{\mu}=(0, \mathbf{d})$ (where $|\mathbf{d}| \simeq \theta$ ), and using the gauge conditions, we have:

$$
k_{\mu} X_{-}^{\mu}=m \omega_{L}\left[\frac{1}{2} \theta^{2} \sigma_{-}-\frac{1}{2} \mathbf{n} \cdot \mathbf{a}^{\prime \prime} \sigma_{-}^{2}+\frac{1}{6}\left(\mathbf{a}^{\prime \prime 2}-\mathbf{d} \cdot \mathbf{a}^{\prime \prime \prime}\right) \sigma_{-}^{3}\right]
$$

together with a similar expression involving $\mathbf{b}$ and $\sigma_{+}$. In the last bracket, the $\mathbf{d} \cdot \mathbf{a}^{\prime \prime \prime}$ term is subdominant, being of order $\mathcal{O}\left(\theta\left|\mathbf{a}^{\prime \prime}\right|^{2}\right)$.

The two integrals in the energy momentum therefore take the form:

$$
I^{\mu}=\int\left[k^{\mu}-d^{\mu}+\ddot{X}^{\mu} \sigma\right] \exp \left[-\frac{i m \omega_{L}}{12}\left(3 \theta^{2} \sigma-3 \theta|\ddot{X}| \sigma^{2} \cos \beta+|\ddot{X}|^{2} \sigma^{3}\right)\right] d \sigma
$$


where $\beta$ is the angle between $\mathbf{d}$ and $\mathbf{a}^{\prime \prime}$. As Damour and Vilenkin pointed out, the first $k^{\mu}$ term is a pure gauge, however, when $d^{\mu} \neq 0$, it cannot be gauged away, as the product $k^{\mu} d_{\mu} \neq 0$, but the trace reversed $\bar{h}_{\mu \nu}$ must be tracefree. However, since correcting for this simply introduces a subdominant term with the same waveform as the main part of the perturbation, like DV, we simply focus on the main part of the integral and compute the main contribution to the waveform. Thus, rewriting

$$
u=\left(\frac{m \omega_{L}}{12} \ddot{X}^{2}\right)^{1 / 3} \sigma \quad, \quad \varepsilon=\left(\frac{m \omega_{L}}{12 \ddot{X}}\right)^{1 / 3} \theta
$$

the relevant part of the integral becomes

$$
I=\left(\frac{12}{m \omega_{L} \ddot{X}^{2}}\right)^{2 / 3} \ddot{X} \int d u(u-\varepsilon) \exp \left[-i\left((u-\varepsilon)^{3}+\varepsilon^{3}+3 \varepsilon u^{2}(1-\cos \beta)\right)\right] .
$$

For $\varepsilon \ll 1$, this integral is well approximated by the $\varepsilon=0$ value:

$$
I_{ \pm}^{\mu}=-\left(\frac{12}{m \omega_{L} \ddot{X}_{ \pm}^{2}}\right)^{2 / 3} \frac{i}{\sqrt{3}} \Gamma\left(\frac{2}{3}\right) \ddot{X}_{ \pm}^{\mu}
$$

and for $\varepsilon>1$, the integral rapidly tends to zero due to the oscillatory behaviour of the term proportional to $(1-\cos \beta)$. Thus DV obtain the logarithmic cusp waveform:

$$
h^{\text {cusp }}(f, \theta) \sim \frac{G \mu L^{2 / 3}}{r|f|^{1 / 3}} H\left[\theta_{m}-\theta\right]
$$

where $H$ is the Heaviside step function, and $\theta_{m}$ is the critical value of $\theta$ for which the integral drops to zero:

$$
\theta_{m}=\left(\frac{12 \ddot{X}}{m \omega_{L}}\right)^{1 / 3} \simeq\left(\frac{2}{L f}\right)^{1 / 3}
$$

using $\ddot{X} \sim 2 \pi / L$, and $f=m \omega_{L} / 2 \pi$.

To transform this to the cosmological setting, one essentially replaces $f$ with $(1+z) f$, where $z$ is the redshift at the time of emission of the cusp GWB, and we must replace $r$ by the physical distance

$$
a_{0} r=a_{0} \int_{t_{e}}^{t_{0}} \frac{d t}{a}=\int_{0}^{z} \frac{d z}{H}=(1+z) D_{A}(z)
$$

where $D_{A}(z)$ is the angular diameter distance at redshift $z$.

Damour and Vilenkin next use the one scale model of a string network, by writing

$$
L \sim \alpha t \quad, \quad n_{L}(t) \sim 1 /\left(\alpha t^{3}\right)
$$


for the length and number density of the string network at cosmological time $t$. Here $\alpha \sim \Gamma G \mu$ is a numerically determined constant, [21, 22, 23], presumed to represent the rate of energy loss from string loops via gravitational radiation. As in DV, we will take $\Gamma \sim 50$, however see [42] for more recent work and discussion on this issue.

Finally, DV estimate the rate of GWB's observed around frequency $f$ coming from the spacetime volume in redshift interval $d z$ :

$$
d \dot{N} \sim \frac{\nu(z)}{(1+z)} \frac{\pi \theta_{m}^{2}(z) D_{A}(z)^{2}}{(1+z) H(z)} d z
$$

where the first factor of $(1+z)$ comes from the redshift of time between emission and observation, $\nu(z)$ is the number of cusp events per unit spacetime volume, and the final part is the measure of the spacetime volume within the beaming cone at redshift $z$, where the beaming cone angle at redshift $z$ is simply given by

$$
\theta_{m}(z)=\left(\frac{2}{(1+z) f L(z)}\right)^{1 / 3} .
$$

The number of cusp events is given by

$$
\nu \sim \mathcal{C} \frac{n_{L}}{P T_{L}} \sim \frac{2 \mathcal{C}}{P \alpha^{2} t^{4}}
$$

where $\mathcal{C}$ is the average number of cusps per loop period $T_{L}=L / 2 \sim \alpha t / 2$ and $P$ is the reconnection probability of the strings. Classical strings which intersect almost always intercommute, thus $P=1$ [25], however the existence of extra dimensions makes it easier for the strings to miss each other. This results in the reconnection probability $P$ being reduced, as strings which appear to meet in 3 dimensions could be missing each other in the extra dimensions, leading to an enhancement of the number density of loops in the string network [32, 12. More detailed simulations, however indicate that this result may be slightly modified [40].

The final step of the DV argument is to integrate (3.15) to find the rate

$$
\dot{N}=\int_{0}^{z_{*}} \frac{d \dot{N}}{d \ln z} d \ln z \sim \frac{d \dot{N}\left(z_{*}\right)}{d \ln z}
$$

and then substituting in a fiducial frequency and desired rate to find the redshift which dominates the signal. Evaluating the gravitational wave at this redshift and frequency then gives the amplitude.

We now revisit this argument with the addition of the effects of the internal extra dimensions.

\section{Wave form in extra dimensions}

In computing the waveform with extra dimensions, there are several features we need to consider [37]. First, there is the motion of the string in the extra dimensions, as 
pointed out by Avgoustidis and Shellard [38], which causes the strings to appear to slow down in our noncompact space dimensions. Next, there is the impact of this motion on the formation of cusps: as we will see, the effect of extra degrees of freedom allows the left and right moving modes to misalign in momentum space, thus avoiding an exact cusp in a similar way to avoiding intercommutation. Finally, there is the gravitational aspect of the extra dimensions. Since these strings are formed in brane inflation scenarios, we will assume that the flux stabilization procedure that prevents dangerous cosmological moduli evolution also prevents the strings from exciting internal degrees of freedom. Thus, we can use the normal 4D gravitational propagator in calculating the gravitational radiation from a cusp.

\subsection{String kinematics with extra dimensions}

We begin with an overview of string solutions in $4+n$ dimensions. As with 4 dimensions, these can be expressed in the Kibble-Turok notation

$$
\mathbf{R}=\frac{1}{2}\left[\mathbf{A}\left(\sigma_{-}\right)+\mathbf{B}\left(\sigma_{+}\right)\right]
$$

where we use upper case to denote the full $3+n$-dimensional spatial vectors, and lower case the noncompact dimensions. As before, $\left|\mathbf{A}^{\prime}\right|^{2}=\left|\mathbf{B}^{\prime}\right|^{2}=1$, hence $\mathbf{A}^{\prime}$ and $\mathbf{B}^{\prime}$ trace out closed curves on a unit $S^{2+n}$. Unlike in 3 space dimensions, where two curves on an $S^{2}$ will generically cross, these curves will generically miss each other. This means that the probability of an exact cusp with extra dimensions is precisely zero. However, from the calculation of the GWB waveform, it is clear that the power is radiated not exclusively from the cusp, but from a region in which the extrinsic curvature of the worldsheet is significant (we will see shortly how the beaming cone opening angle defines this).

We therefore generalise the exact cusp to a "near cusp event" (NCE) for which

$$
\left|\mathbf{A}^{\prime}-\mathbf{B}^{\prime}\right|=2 \Delta
$$

where $\Delta \ll 1$ is a parameter measuring how close to an exact cusp (EC) we are. We can visualise the near cusp event as a rounded cusp, as indicated in figure $\mathbb{1}$.

In order to estimate the probability of near cusp formation, we first assume an even measure in parameter space (we will discuss alternative possibilities later). Each loop carries left and right moving waves of harmonics of the fundamental frequency mode $2 \pi / L$, the wave vectors of which are constrained by the higher dimensional version of the gauge restriction (2.9). These can be represented in terms of the rotation group $S O(n+3)$ 43], and thus the parameter space of the loop is simply parametrized by a set of angles. An example of some low harmonic loops with one periodic extra dimension analogous to those considered by Kibble and Turok [26] is given presently. These show how the compactification of the extra dimension makes little difference to the self-intersection probability for a zero width string, and demonstrates nicely the cusp rounding effect. 
We estimate the probability of NCE's therefore as $g(\Delta)^{q}$, where $q$ is the codimension in parameter space of the subspace formed by loops which contain exact cusps, and $g$ is a function which relates a shift in a parameter to a change in $\left|\mathbf{A}^{\prime}-\mathbf{B}^{\prime}\right|$. $q$ can be readily computed from the condition for a cusp:

$$
\mathbf{A}^{\prime}=\mathbf{B}^{\prime}
$$

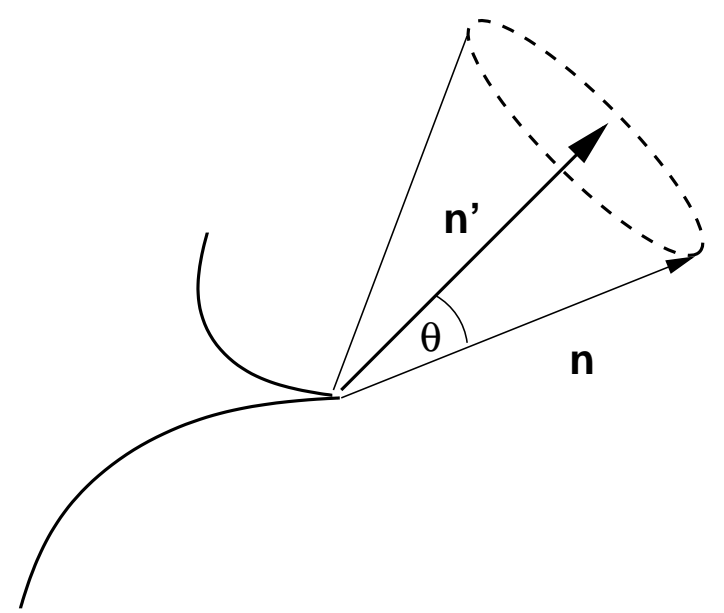

This is a set of $n+3$ equations, however, as $\left|\mathbf{A}^{\prime}\right|=\left|\mathbf{B}^{\prime}\right|=1$ this results in $n+2$ constraints. Of these, two are used to fix the values of $\sigma_{ \pm}$at the cusp, hence $n$ constraints in parameter space remain. Thus, the codimension of the exact cusp space is precisely the number of extra dimensions $q=n$. In order to determine $g$, we modelled explicit loop solutions with one extra dimension, and found that $g(\Delta)=g_{0} \Delta$, where $g_{0} \simeq 1$ : see figure 2, where we plot $\Delta=\min \mid \mathbf{A}^{\prime}-$

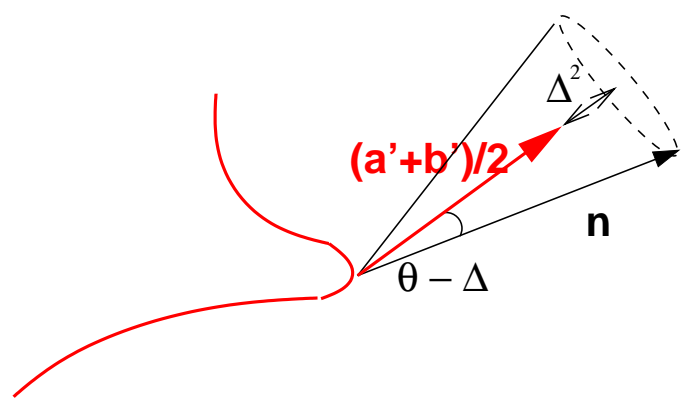

Figure 1: A sketch of a near cusp event as opposed to an exact cusp. The rounding of the cusp is indicated, as is the narrowing of the beaming cone.

The outcome of our analysis is therefore that the number of NCE's with $\mid \mathbf{A}^{\prime}-$ $\left.\mathbf{B}^{\prime}\right|_{\min } \leq 2 \Delta$ in a generic loop is $\mathcal{N}(\Delta) \simeq \Delta^{n}$ (since all loops have $\left|\mathbf{A}^{\prime}-\mathbf{B}^{\prime}\right| \leq 2$ at all points on their trajectory). This argument of course simply refers to the cusps in the full higher dimensional loop motion, and not those loops which are close to our 3-dimensional loops; it also makes no reference to any warping of any of the spacetime dimensions. In addition, it assumes an exact Nambu description, i.e. an exactly zero width string. The strings will in general have finite width, and we expect that should the string width become a significant fraction of the internal dimension size, then the motion in the internal dimension will be irrelevant. Note however, that because these strings are basically classical objects, there is no quantization of the motion in the internal directions.

A nice example of the effects of extra dimensions is given by constructing a loop family. The general solution for the left moving half for example is given by:

$$
\mathbf{A}\left(\sigma_{-}\right)=\sum_{n} \frac{L}{2 \pi n} \mathbf{C}_{n} \sin \left(\frac{2 \pi n \sigma_{-}}{L}\right)+\frac{L}{2 \pi n} \mathbf{D}_{n} \cos \left(\frac{2 \pi n \sigma_{-}}{L}\right)
$$



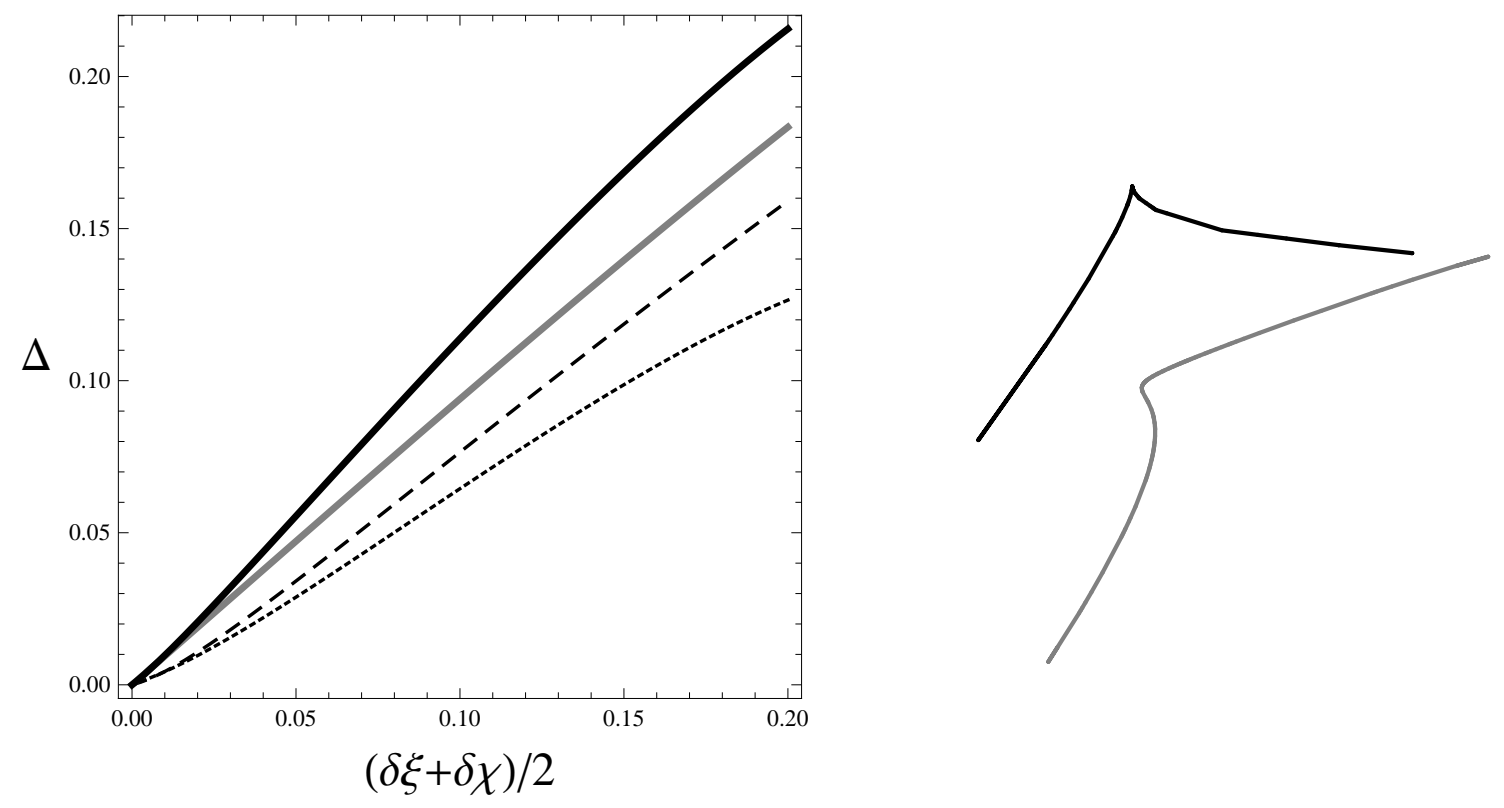

Figure 2: An examination of the dependence of the near cusp parameter, $\Delta$, on the loop solution parameters for the 1-5/1 loop family given in (4.7,4.9). The magnitude of $\left|\mathbf{A}^{\prime}-\mathbf{B}^{\prime}\right|$ is computed as a function of the loop parameters as we move away from an exact cusp event in parameter space. The 'normal' direction in solution space was computed using an expansion around the exact cusp. The approximate linearity of the relation is demonstrated for a range of parameter values and initial cusp values. An exact cusp occurs when $\xi=-\chi, \zeta=\phi_{1}=-\phi_{2}$. The black solid line corresponds to $\xi=\pi / 4, \zeta=\pi / 6$, the grey line to $\xi=\pi / 3, \zeta=\pi / 4$, the dashed line to $\xi=\pi / 4, \zeta=\pi / 5$, and the dotted line to $\xi=\pi / 4, \zeta=\pi / 12$. The plot on the right shows the effect of moving away from the cusp of the black parameter family by a parameter shift of 0.1 .

where the gauge conditions imply

$$
\begin{aligned}
2 & =\sum_{n, m}\left(\mathbf{C}_{n} \cdot \mathbf{C}_{m}-\mathbf{D}_{n} \cdot \mathbf{D}_{m}\right) \cos \frac{2 \pi(n+m) \sigma_{-}}{L}+\left(\mathbf{C}_{n} \cdot \mathbf{C}_{m}+\mathbf{D}_{n} \cdot \mathbf{D}_{m}\right) \cos \frac{2 \pi(n-m) \sigma_{-}}{L} \\
& +\left(\mathbf{C}_{n} \cdot \mathbf{D}_{m}+\mathbf{D}_{n} \cdot \mathbf{C}_{m}\right) \sin \frac{2 \pi(n+m) \sigma_{-}}{L}+\left(\mathbf{C}_{m} \cdot \mathbf{D}_{n}-\mathbf{C}_{n} \cdot \mathbf{D}_{m}\right) \sin \frac{2 \pi(n-m) \sigma_{-}}{L}(4.5)
\end{aligned}
$$

A simple example of a new solution when we have one extra dimension is to choose two independent harmonics $n>m$, with $n \neq 3 m$ (so that $2 n, n+m, n-m$, and $2 m$ are all distinct). The constraints from (4.5) give

$$
\begin{gathered}
\mathbf{C}_{n} \cdot \mathbf{D}_{n}=\mathbf{C}_{m} \cdot \mathbf{D}_{m}=\mathbf{C}_{n} \cdot \mathbf{C}_{m}=\mathbf{D}_{n} \cdot \mathbf{D}_{m}=\mathbf{C}_{n} \cdot \mathbf{D}_{m}=\mathbf{C}_{m} \cdot \mathbf{D}_{n}=0 \\
\mathbf{C}_{n}^{2}=\mathbf{D}_{n}^{2} \quad ; \quad \mathbf{C}_{m}^{2}=\mathbf{D}_{m}^{2} \\
\mathbf{C}_{n}^{2}+\mathbf{D}_{n}^{2}+\mathbf{C}_{m}^{2}+\mathbf{D}_{m}^{2}=2 .
\end{gathered}
$$


Thus we can take

$$
\mathbf{C}_{m}=\cos \zeta \mathbf{e}_{1}, \quad \mathbf{C}_{n}=\sin \zeta \mathbf{e}_{2}, \quad \mathbf{D}_{m}=\cos \zeta \mathbf{e}_{3}, \quad \mathbf{D}_{n}=\sin \zeta \mathbf{e}_{4} .
$$

Clearly this solution, with its requirement of 4 mutually orthogonal vectors, is a simple example of a new solution in higher dimensions. In three space dimensions (3D), we can only have a single harmonic, unless $n=3 \mathrm{~m}$. Thus the $3 \mathrm{D}$ limit of this left moving half is $\zeta=0, \pi / 2$. To give an illustrative loop family, we will take the right moving half to have a single harmonic only

$$
\mathbf{B}\left(\sigma_{+}\right)=\frac{L}{2 \pi} \mathbf{v}_{1} \sin \left(\frac{2 \pi \sigma_{+}}{L}\right)+\frac{L}{2 \pi} \mathbf{v}_{2} \cos \left(\frac{2 \pi \sigma_{+}}{L}\right)
$$

where $\mathbf{v}_{1}$ and $\mathbf{v}_{2}$ are two mutually orthogonal vectors, which will be given by an $\mathrm{SO}(4)$ rotation of the $(x, y)$ plane:

$$
\mathbf{v}_{1}=\left(\begin{array}{c}
\cos \xi \cos \chi \cos \phi_{1}-\sin \xi \sin \chi \cos \phi_{2} \\
\cos \xi \cos \chi \sin \phi_{1}+\sin \xi \sin \chi \sin \phi_{2} \\
\sin \xi \cos \chi \cos \left(\phi_{1}+\phi_{2}\right)+\cos \xi \sin \chi \\
-\sin \xi \cos \chi \sin \left(\phi_{1}+\phi_{2}\right)
\end{array}\right) ; \mathbf{v}_{2}=\left(\begin{array}{c}
\sin \xi \sin \chi \sin \phi_{2}-\cos \xi \cos \chi \sin \phi_{1} \\
\cos \xi \cos \chi \cos \phi_{1}+\sin \xi \sin \chi \cos \phi_{2} \\
-\sin \xi \cos \chi \sin \left(\phi_{1}+\phi_{2}\right) \\
\cos \xi \sin \chi-\sin \xi \cos \chi \cos \left(\phi_{1}+\phi_{2}\right)
\end{array}\right)
$$

This loop family corresponds to a $m-n / 1$ string in the notation of [27].

A time sequence of an evolving loop is shown in figure 3, where we have taken $m=1, n=5$ to be specific, and set $L=2 \pi$ for convenience. A generic solution is compared with the $3 \mathrm{D}$ solution with only one harmonic.

\subsection{The gravitational waveform}

We now compute the waveform for a NCE with parameter $\Delta$. The main difference between the EC and the NCE is that the velocity $\dot{X}^{\mu}=\left(1,\left(\mathbf{a}^{\prime}+\mathbf{b}^{\prime}\right) / 2\right)$ is now no longer necessarily null, and that the individual left and right moving velocities need not be aligned. In other words, in evaluating the integral (3.7), we no longer have " $\varepsilon=0$ ", since there are additional phase terms coming from the misalignment of $\mathbf{a}^{\prime}$ and $\mathbf{b}^{\prime}$, as well as from the fact that $\dot{X}_{ \pm}^{\mu}$ is no longer null.

Define

$$
\begin{aligned}
\boldsymbol{\delta} & =\frac{1}{2}\left(\mathbf{a}^{\prime}-\mathbf{b}^{\prime}\right) \\
\mathbf{n}^{\prime} & =\frac{\left(\mathbf{a}^{\prime}+\mathbf{b}^{\prime}\right)}{\left|\mathbf{a}^{\prime}+\mathbf{b}^{\prime}\right|}
\end{aligned}
$$

to be the separation vector of $\mathbf{a}^{\prime}$ and $\mathbf{b}^{\prime}$ at the $\mathrm{NCE}$, and the direction vector of the NCE respectively. Then writing $\mathbf{A}^{\prime}=\left(\mathbf{a}^{\prime}, \boldsymbol{a}\right), \mathbf{B}^{\prime}=\left(\mathbf{b}^{\prime}, \boldsymbol{b}\right)$, shows that $\left|\mathbf{a}^{\prime}\right|^{2}=1-a^{2}$, and $\left|\mathbf{b}^{\prime}\right|^{2}=1-b^{2}$ (with $a=|\boldsymbol{a}|$ etc.). A quick check of (3.5), (3.6) then indicates that the gravitational integral (3.7) will be damped unless $a, b \ll 1$. While our 

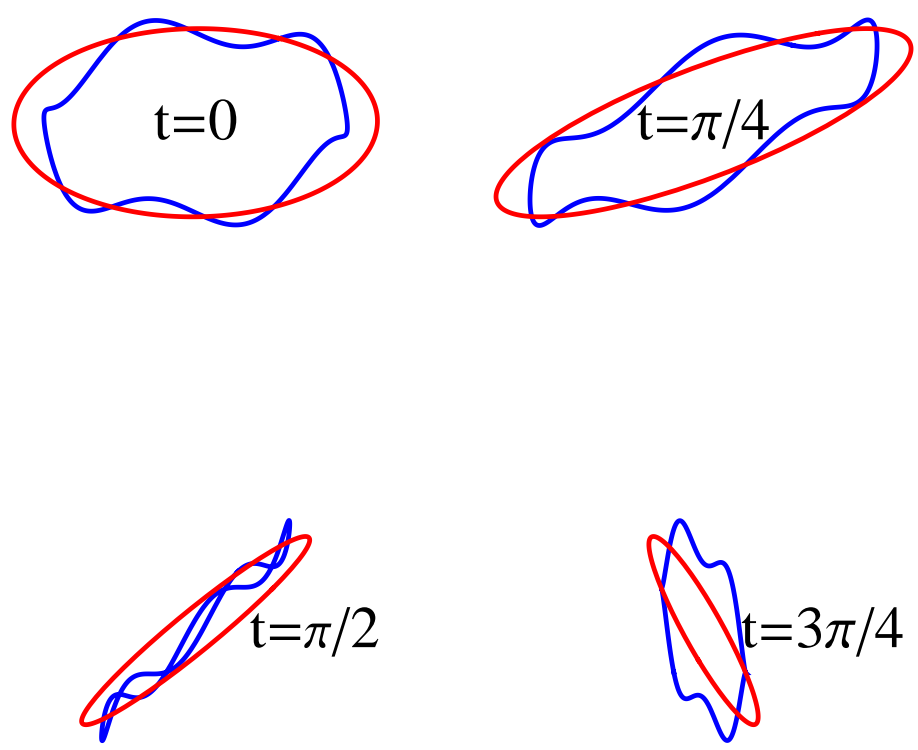

Figure 3: The time evolution of a 1-5/1 harmonic string with generic parameters $\xi=$ $-\chi=\pi / 3, \phi_{1}=-\phi_{2}=\pi / 4$ and $\zeta=\pi / 4$ in blue, and the 3D loop with $\zeta=0$ in red.

modelling with compact extra dimensions indicates no particular restrictions on the parameter space, the expectation is that either warping of extra dimensions, or some other kinematic consequence of cosmological expansion, will lead to the trajectories being somehow close to the four dimensional behaviour (although [44] indicates this may not be the case). We will therefore take $a, b \ll 1$ from now on. Under these assumptions, expansion of $\boldsymbol{\delta}$ and $\mathbf{n}^{\prime}$ gives generically that $a^{2} \sim b^{2}=\mathcal{O}\left(\Delta^{2}\right)=\mathcal{O}(\delta)$. Thus in orders of magnitude

$$
\begin{aligned}
& \mathbf{a}^{\prime}=\frac{1}{2}\left|\mathbf{a}^{\prime}+\mathbf{b}^{\prime}\right| \mathbf{n}^{\prime}+\boldsymbol{\delta} \simeq\left(1-\frac{\Delta^{2}}{2}\right) \mathbf{n}^{\prime}+\boldsymbol{\delta} \\
& \mathbf{b}^{\prime}=\frac{1}{2}\left|\mathbf{a}^{\prime}+\mathbf{b}^{\prime}\right| \mathbf{n}^{\prime}-\boldsymbol{\delta} \simeq\left(1-\frac{\Delta^{2}}{2}\right) \mathbf{n}^{\prime}-\boldsymbol{\delta} .
\end{aligned}
$$

Finally, estimating $\mathbf{n}^{\prime} . \mathbf{a}^{\prime \prime} \sim \mathbf{n}^{\prime} \cdot \mathbf{b}^{\prime \prime}=\mathcal{O}(\Delta)|\ddot{X}|$ we find (making the same approximations as DV) the expression

$$
k_{\mu} X_{-}^{\mu}=\frac{1}{2}\left(\theta^{2}+\Delta^{2}\right) \sigma_{-}-\frac{1}{2}(\theta+\Delta)|\ddot{X}| \sigma_{-}^{2}+\frac{1}{6} \ddot{X}^{2} \sigma_{-}^{3}
$$

with a similar expression involving $X_{+}$and $\sigma_{+}$. 
Thus we find that the waveform of the NCE is the same as that of the EC, with the proviso that the cone opening angle is now decreased to

$$
\theta_{\Delta}=\theta_{m}-\Delta \simeq\left(\frac{2}{L f}\right)^{1 / 3}-\Delta
$$

i.e. the (logarithmic) NCE waveform is

$$
h^{\mathrm{NCE}} \sim \frac{G \mu L^{2 / 3}}{r|f|^{1 / 3}} H\left[\theta_{\Delta}-\theta\right] .
$$

Notice that (4.15) provides a high frequency cutoff to the waveform,

$$
f_{\Delta}=1 /\left(\Delta^{3} T_{L}\right)
$$

therefore our long frequency 'tail' to the waveform is curtailed at some (high) $\Delta$ dependent frequency. However, what is more relevant cosmologically is the combination of the impact of this cutoff of the beaming cone area and the effect of the lowering of the number of NCE's.

Cosmologically, we need to calculate the GWB event rate $\dot{N}$ for near cusp events, however, for a general network there will be a range of NCE's with different $\Delta$ values, up to and including the cutoff value when the GWB beaming cone closes off. We clearly need to integrate over these options to obtain the nett effect of all possible NCE's. We therefore write

$$
\frac{d^{2} \dot{N}_{\mathrm{NCE}}}{d z d \Delta} \sim \frac{\mathcal{C}(\Delta) n_{L}(z)}{P T_{L}(z)} \frac{\pi\left(\theta_{m}(z)-\Delta\right)^{2} D_{A}(z)^{2}}{(1+z)^{2} H(z)}
$$

where $\mathcal{C}(\Delta)$ is the local probability density of NCE's for the network. In four spacetime dimensions, a loop with continuous momentum functions always has a cusp, which would correspond to $\mathcal{C}(\Delta)=\delta(\Delta)$ in an integration of (4.18) (where $\delta(\Delta)$ is now the Dirac $\delta$-function!). For extra dimensions, assuming that the loops are spread evenly in the parameter space of solutions, we get

$$
\mathcal{C}(\Delta)=\mathcal{N}^{\prime}(\Delta)=n \Delta^{n-1}
$$

and hence the $\Delta$ integral yields

$$
\int_{0}^{\theta_{m}} \mathcal{C}(\Delta)\left(\theta_{m}(z)-\Delta\right)^{2}=\frac{2 \theta_{m}(z)^{n+2}}{(n+1)(n+2)}
$$

where the integral is saturated by $\theta_{m}<1$. Note that for the fiducial frequency $f \sim 150 \mathrm{~Hz}, \theta_{m} \sim 10^{-4} \rightarrow 10^{-2}$ as $G \mu \sim 10^{-6} \rightarrow 10^{-12}$ respectively, and since $\theta_{m}$ varies as $(1+z)^{1 / 6}$, it remains small until extremely high redshifts $\left(\left(1+z_{\mathrm{rec}}\right)^{1 / 6} \simeq 3\right)$. 
Gathering together these different effects, we therefore arrive at the expression for the GWB rate:

$$
\frac{d \dot{N}_{\mathrm{NCE}}}{d z}=\frac{2 \theta_{m}(z)^{n+2}}{(n+1)(n+2)} \frac{n_{L}(z)}{P T_{L}(z)} \frac{\pi D_{A}(z)^{2}}{(1+z)^{2} H(z)} .
$$

Figure 4 shows the gravitational wave amplitude for the cosmic string cusp bursts in the form presented by DV, 32], for varying values of $n$. The graphs are presented first by calculating the amplitude in exactly the way DV did, by using interpolating functions, and also neglecting $\Omega_{\Lambda}$. However, the dotted data curves also show an exact redshift integration, keeping the precise values of the angular diameter and cosmological time for the concordance cosmology $\left(\Omega_{r}=4.6 \times 10^{-5}, \Omega_{m}=0.28, \Omega_{\Lambda}=\right.$ $\left.1-\Omega_{m}-\Omega_{r}\right)$ and integrating out numerically in redshift space for different values of $G \mu$. A similar plot is obtained for the LISA frequency band, [37], however, the suppression of the signal is less marked at lower frequency.

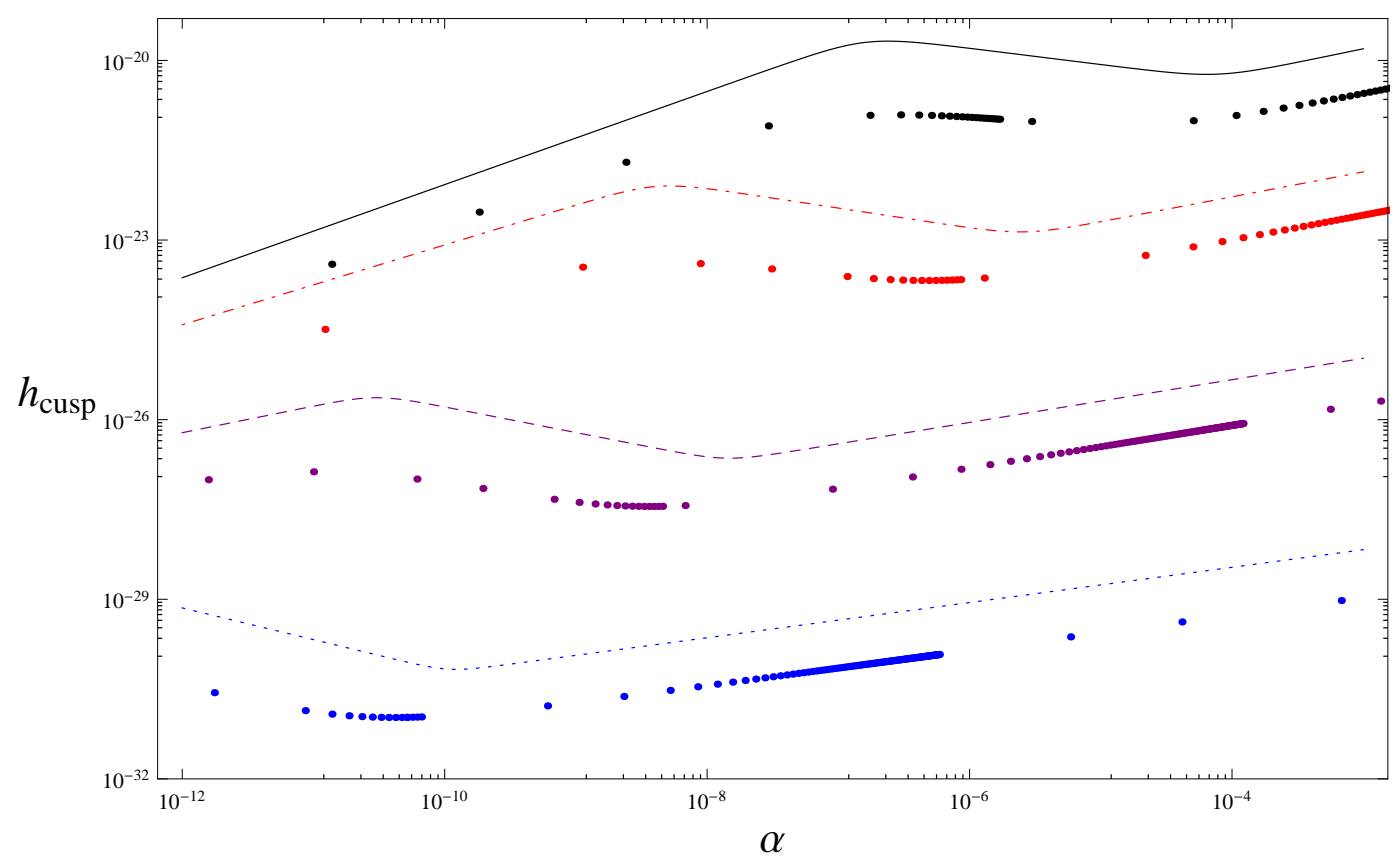

Figure 4: A log log plot of the GW amplitude of bursts as a function of $\alpha$ for a fiducial frequency $f=150 \mathrm{~Hz}$, and a detection rate of 1 per year. The lines (solid or dotted/dashed) represent the graphs obtained using the DV interpolating functions, allowing for a direct comparison with [32]. The sets of individual dots correspond to the exact numerical redshift integrations, where we used the exact functions $t(z), D_{A}(z)$, for the concordance cosmology. The plots are colour coded, from the black, DV result at the top, through red (dot-dash) for $n=1$, purple (dashed) for $n=3$, and blue (dotted) for $n=6$ and all have an intercommutation probability of $P=10^{-3}$.

An alternative way of presenting the GWB information is to instead compute the expected detection rate of events with amplitudes greater than (or equal to) a 


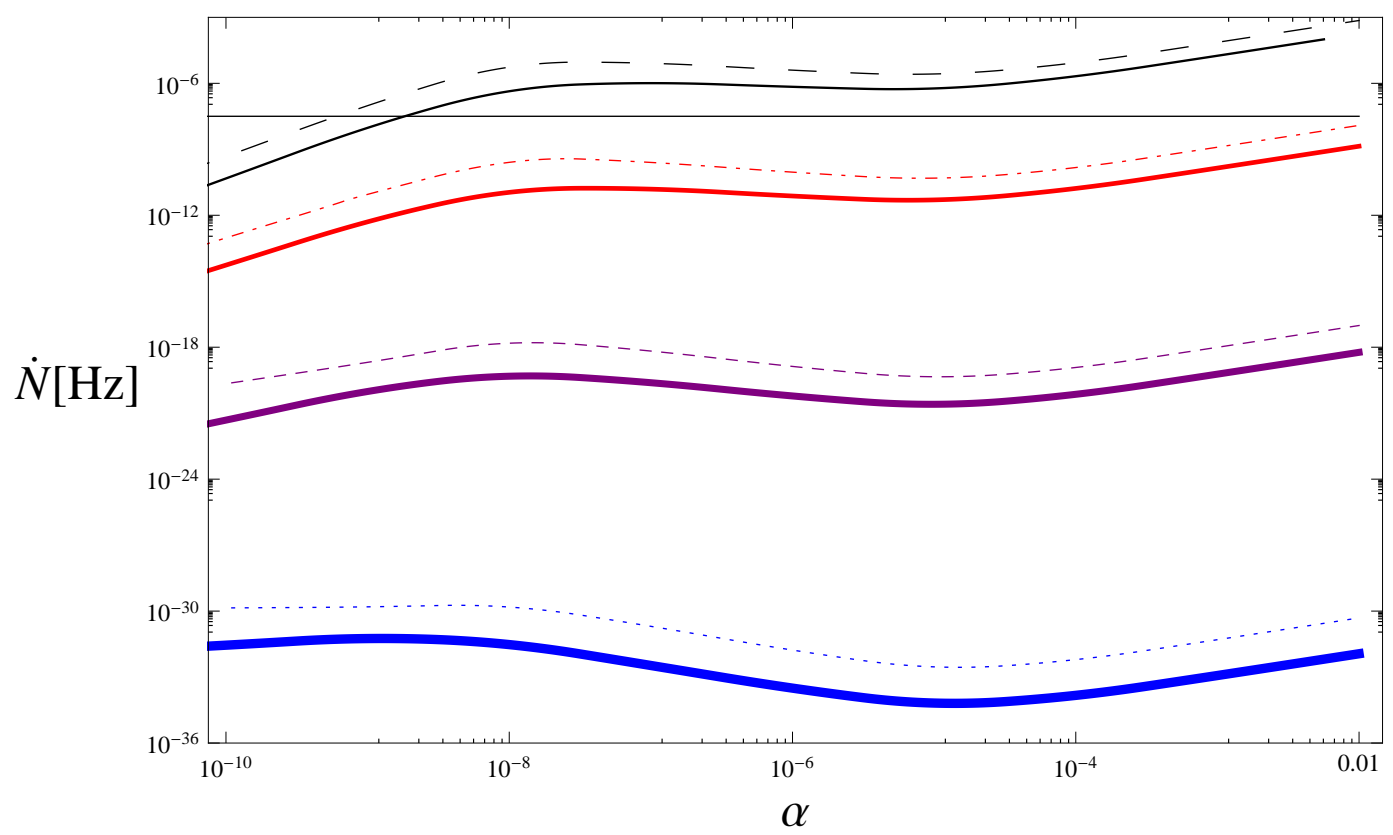

Figure 5: A similar plot to that of figure 4, but in this case showing the expected rate at an amplitude of $10^{-21} s^{-1 / 3}$, using the method of [33]. The plots are colour coded as in figure 1 except in this case the thick solid lines are now the numerical integration results. From top to bottom: the 3D $P=10^{-3}$ result, and the extra dimension plots with $n=1$, $n=3$, and $n=6$ respectively. The horizontal black line indicates a rate of one event per year.

given amplitude. As explained in the papers of Siemens et al. [33, 34], the one scale model used by DV, in which all loops are taken to have essentially the same length (3.14), does not capture the full dynamical range of the cosmic string network which will have loops, in theory, at all scales. They therefore recomputed the rate in order to take into account the dependence not only on redshift, but also on length scales, which they encoded in the amplitude of the cusp waveform $A$, found by considering

$$
h^{c u s p}=A|f|^{-1 / 3}
$$

(in the logarithmic representation of DV) and comparing with (3.11), or in the extra dimensional case, (4.16), resulting in a rate per redshift interval $d z$ and amplitude interval $d A$, rather than the rate per redshift interval obtained by DV. However, on using the one scale model (3.14), where amplitudes are directly associated with redshifts, the amplitude dependence is effectively integrated out and the generalised expression found in 33 reduces to (3.15), the expression used by DV and hence to (4.21) in the extra dimensional case. The results in 33] are therefore presented as a rate plot against the string parameter $G \mu$, calculated by integrating $d \dot{N}$ out to redshift values corresponding to the chosen amplitude (we use their value of $A=$ 
$10^{-21} \mathrm{~s}^{-1 / 3}$ only) at various values of $G \mu$ :

$$
\frac{\varphi_{t}^{2 / 3}(z)}{(1+z)^{1 / 3} \varphi_{r}(z)}=\frac{50 A H_{0}^{-1 / 3}}{\alpha^{5 / 3}}
$$

where $\alpha \sim 50 G \mu$ as usual, and $\varphi_{t}, \varphi_{r}$ are either the DV interpolating functions, or are related to the exact functions $t(z), D_{A}(z)$ (c.f. Siemens Appendix A [33]). This equation relating the redshift and amplitude is easily found from the expression for the cusp waveform derived in [31]. Figure 5 shows the rates calculated for the 3D and extra dimensional cases using this alternative approach.

\section{Discussion}

The clear message of our results is that the impact of motion in extra dimensions can be significant. That extra dimensions should have an effect is not unreasonable, since they can be viewed as additional degrees of freedom living on the string $^{3}$, for example, superconducting cosmic strings, [45], can be represented as a dimensional reduction of standard five dimensional KK theory [46]. For these cosmic strings, the currents round off cusps in much the same way as we have described here, [47], and also alter the balance between the energy and tension of the string, [48, which has a clear gravitational impact. This naturally raises the concern that we may not be able to distinguish between extra fields living on a cosmic string, and extra dimensions in which the string is moving. This would certainly be the case if one was observing a string and a single GWB. However, our calculation was for the expected signal from a cosmological network of strings, which depends not only on the GWB waveform, but also on the properties of the network. Superconducting string networks have not been as well explored as those of standard cosmic strings, 49, with the main focus being on the different physics induced by the long range electromagnetic interactions. Nevertheless, as superconducting strings have similar intercommutation properties to standard cosmic strings [50], it is likely that the network is more similar to the usual cosmic string network than that of the cosmic superstrings. Thus, while the individual GWB waveforms will be similar, the expected rates and signals we have calculated for the cosmological networks are indeed specific to extra dimensions.

It is perhaps a little surprising that the effect of extra dimensions can be so dramatic. We therefore now examine our assumptions carefully, raising below a series of critiques together with a discussion of their validity and resolution.

The basic reason for the suppression of the signal is the distribution over the near cusp parameter $\Delta$. Our simulations with test loop trajectories were performed in flat space with a toroidal (flat) compactification. Clearly the cosmological evolution will influence the distribution of momentum across the various modes (although paradoxically it would seem to damp higher momenta in the noncompact dimensions).

\footnotetext{
${ }^{3}$ We thank Jose Blanco-Pillado for discussions on this point.
} 
Since these strings form from the collision of a brane and anti-brane, it seems likely that they have significant initial momentum in the extra dimensions, thus we see no reason to curtail our solution space in this way. The main objection to having total freedom of the internal modes is that by wrapping back and forth across the extra dimension(s) the string has more opportunity to self intersect, thus curtailing the additional freedom in that direction.

We modelled this effect by exploring the self-intersection of a $1-3 / 1$ family of loops. We chose this combination of harmonics, as the $4 \mathrm{D}$ family will have a $3 \mathrm{D}$ limit which were the first simple loop trajectories explored by Kibble and Turok [26]. In 3D, the loop family self intersects approximately $30 \%$ of the time (note, the original plot of Turok [26] is inaccurate, see [51] for the correct version). When an additional dimension is introduced, the measure of the solution set allowing for self intersections again becomes zero: the argument is once more parametric. A string will self-intersect if

$$
\mathbf{A}(\tau-\sigma)+\mathbf{B}(\tau+\sigma)=\mathbf{A}\left(\tau-\sigma^{\prime}\right)+\mathbf{B}\left(\tau+\sigma^{\prime}\right)
$$

for some $\tau, \sigma, \sigma^{\prime}$. Thus, there are three dynamical variables and $3+n$ constraints. In $3 \mathrm{D}$, it is therefore possible to satisfy these constraints simultaneously, although a more careful check of the parameters shows that not all loop solutions can satisfy these constraints. Nonetheless, it shows how the subspace of self intersecting loops can be of nonzero measure in parameter space. With extra dimensions however, satisfying (5.1) necessarily requires a constraint on parameter space, hence the subspace with self intersections will be of lower dimension than parameter space. Even compactifying the extra dimensions does not change this argument, unless we take into account the finite width of the string. Essentially, if we take the string to have zero width, then it can easily miss itself even when winding back and forth across the extra dimensions many times. However, with finite width, the self intersection probability now becomes nonzero, and of order $\mathcal{O}(w / R)^{n}$ (where $w$ is the string width, and $R$ the size of the extra dimension). This therefore suggests that we introduce this ratio in a finite width correction to the GWB measure.

As we mentioned during our initial discussion, warping of the extra dimensions could also provide some significant dynamical effect. The results of Avgoustidis [44] indicate that warping is not as dramatic a trapping force as intially suspected, however, any confinement of strings could be significant, and a detailed modelling of this effect is necessary. For now, we model a restriction in the extra dimensional motion by a restriction in $\Delta$. Instead of allowing $\Delta$ to range over the full unit interval, we restrict $\Delta \in\left[0, \Delta_{0}\right]$. Thus we must renormalize $\mathcal{C}$ :

$$
\mathcal{N}\left(\Delta_{0}\right)=\int_{0}^{\Delta_{0}} \mathcal{C}(\Delta)=1 \quad \Rightarrow \quad \mathcal{C}(\Delta)=\frac{n}{\Delta_{0}^{n}} \Delta^{n-1}
$$


Thus the relevant $\Delta$ integral (4.20) now becomes

$$
\int_{0}^{\min \left\{\Delta_{0}, \theta_{m}\right\}} \mathcal{C}(\Delta)\left(\theta_{m}(z)-\Delta\right)^{2}=\theta_{m}(z)^{2} \mathcal{F}_{n}\left[\frac{\theta_{m}}{\Delta_{0}}\right]
$$

where

$$
\mathcal{F}_{n}[x]=\frac{2 x^{n}}{(n+1)(n+2)} H[1-x]+\left(1-\frac{2 n}{(n+1) x}+\frac{n}{(n+2) x^{2}}\right) H[x-1] .
$$

A reasonable value for $\Delta_{0}$ might be to use the one parameter ratio that does impact on the loop families and motion: the ratio of string width to the size of the extra dimension. The Nambu action is only a good approximation when the width of the string is small compared to scales of physical interest. This is rarely a problem in cosmology, as the string width is set by the inflationary scale, and the size of the universe rapidly becomes large. As far as the extra dimensions are concerned however, these are stabilized at a couple of orders of magnitude above the string scale, hence while the Nambu action is a good approximation, we might expect some corrections to show up due to parameter restriction from self intersection or excessive winding as already discussed. We therefore expect this parameter to be related to the probability of intercommutation, which can be viewed as arising because of the strings "missing" each other in the internal dimensions.

To test this alternate expression, we took values of $\Delta_{0}=0.1,0.01,0.001$, and $10^{-4}$. These values were motivated by a limiting sensible ratio $w / R$, and the value of $P$. From (5.3), we see that the effect of $\Delta_{0}$ is to cut off the integral as $\theta_{m}$ grows. For $\theta_{m}<\Delta_{0}$, the dependence of the rate on $\theta_{m}(z)$ remains that of the previous section, however, as $\theta_{m}$ grows, the functional dependence shifts towards the $\theta_{m}(z)^{2}$ form of the 3D result. From the expression for $\theta_{m}(z)$, (3.16), we see that this is proportional to $(G \mu)^{-1 / 3}$, hence the rates converge to the 3D value sooner for smaller $G \mu$.

Figure 6 shows the effect of the $\Delta_{0}$ parameter on the event rate at an amplitude cutoff of $10^{-21} \mathrm{~s}^{-1 / 3}$ for $\Delta_{0}$ ranging from $10^{-1}-10^{-4}$ as indicated. Note that once we use this more complicated expression (5.3), the use of the interpolating function approximation becomes too unwieldy, and the rates had to be calculated by direct integration. Figure 7 shows the effect of the rate on the number of extra dimensions, fixing $\Delta_{0}=10^{-3}$ and allowing $n$ to vary as indicated. Here we see that for all $n$ the plots converge on the 3D result at $\alpha \sim 10^{-11}$ but for $\alpha \sim 10^{-8}$ for example, the rate drops by roughly an order of magnitude per dimension. A positive detection therefore could potentially tell us the number of extra dimensions!

To sum up: We have included the effect of motion in extra dimensions in the computation of the gravitational wave burst signal from cusp events on cosmic string loops. We find a significant effect, even after taking into account finite width effects and the size of the extra dimension. Clearly further work is required to get better control of the approximations being used, in particular to take into account more 


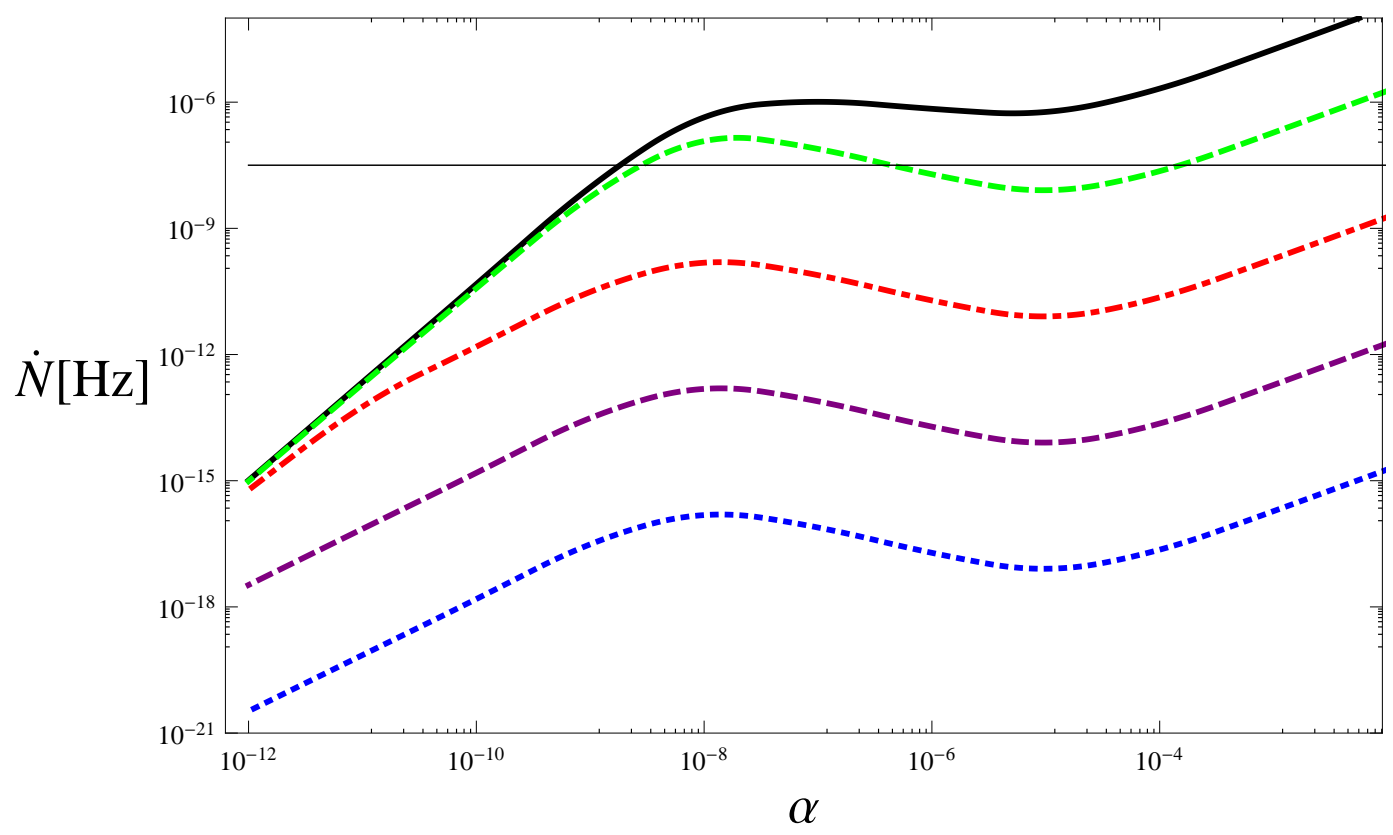

Figure 6: A plot of the dependence of the rate on $\Delta_{0}$ with the number of extra dimensions fixed at three. From top to bottom, the solid black line indicates the $3 \mathrm{D}$ result, $\Delta_{0}=10^{-4}$ in dashed green, $\Delta_{0}=10^{-3}$ in dot-dash red, $\Delta_{0}=10^{-2}$ in dashed purple, and $\Delta_{0}=10^{-1}$ in dotted blue respectively. The horizontal black line indicates a rate of one event per year.

complex compactification geometries, however it does seem that motion in internal dimensions is important. Finally, if the string tension lies in the serendipitous range $10^{-8}-10^{-10}$ then the possibility arises that a positive detection by gravitational radiation would not only confirm the general brane inflation scenario, but could provide a means of determining the number of (effective) extra dimensions.

\section{Acknowledgments}

We would like to thank A. Avgoustidis, J.J. Blanco-Pillado, D. Chung, L. Leblond, S. Rajamanoharan, P. Shellard and G.Shiu for helpful discussions.

RG and IZ would like to thank the Perimeter Institute for hospitality while this work was being undertaken, and RG would like to thank the Cambridge Centre for Theoretical Cosmology for hospitality while this work was being completed.

SC is supported by EPSRC, EOC is supported by EC FP6 through the Marie Curie EST project MEST-CT-2005-021074, GG is suppported by the Government of Canada through Industry Canada and by the Province of Ontario through the Ministry of Research, RG is partially supported by STFC, and IZ is partially supported by the SFB-Transregio TR33 "The Dark Universe" (DFG) and the EU FP7 program PITN-GA-2009-237920. 


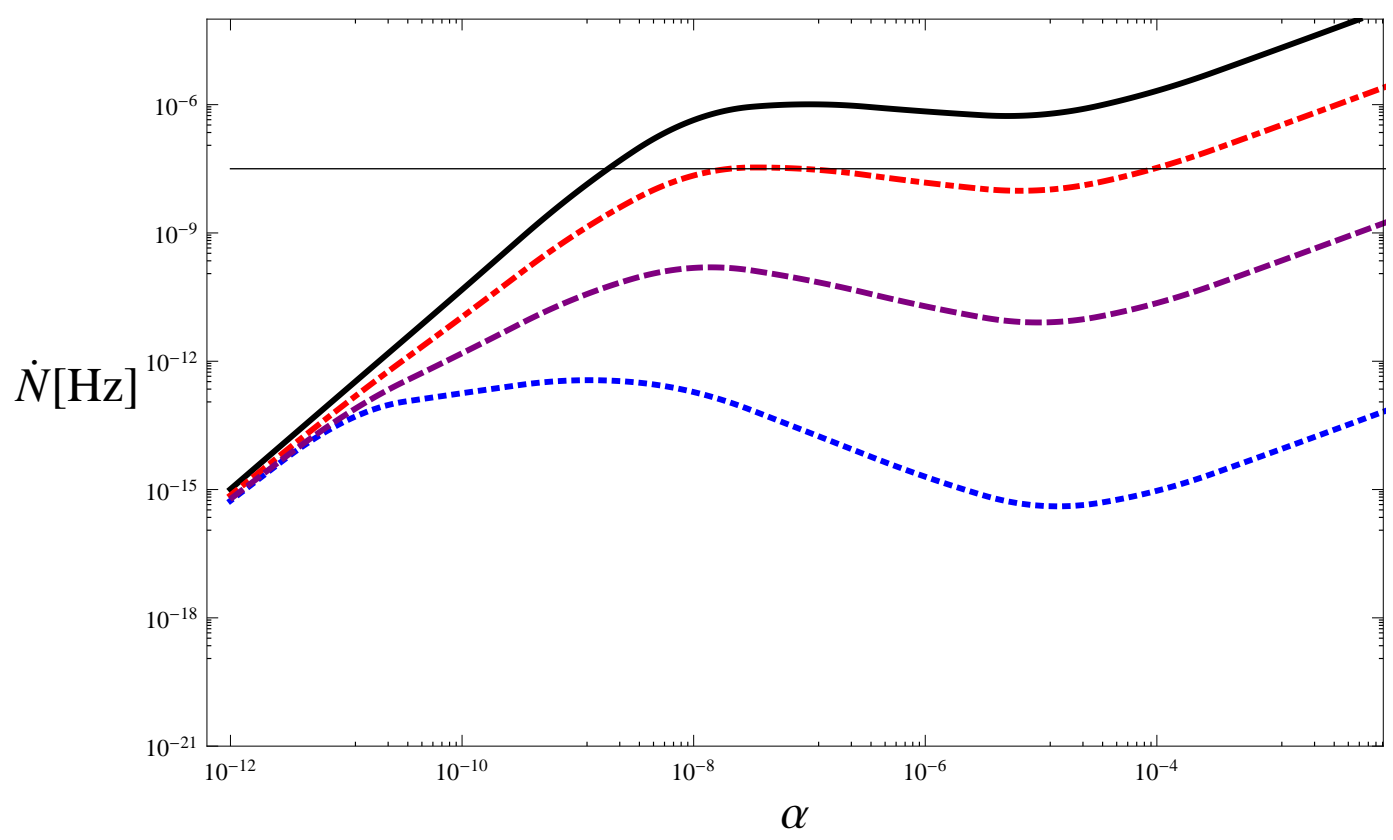

Figure 7: A recomputation of the rate plot with the new nearcusp measure (5.3) fixing $\Delta_{0}=10^{-3}$, and allowing $n$ to vary. From top to bottom, $n=0,1,3$ and 6 respectively. The horizontal black line indicates a rate of one event per year.

\section{A. Finite width corrections to the cusp}

In order to examine the validity of the Nambu approximation, we investigate the behaviour of the extrinsic curvature in the vicinity of an exact cusp. A 2D worldsheet living in 4 dimensions has codimension 2 and thus there exist two families of normals $n_{i}^{\mu}$, satisfying:

$$
\begin{aligned}
n_{i}^{\mu} n_{j}^{\nu} \eta_{\mu \nu} & =-\delta_{i j} \\
n_{i}^{\mu} \frac{\partial X^{\nu}}{\partial \sigma_{A}} \eta_{\mu \nu} & =0 .
\end{aligned}
$$

We choose our normals to be

$$
\begin{aligned}
& n_{1}^{\mu}=\left(1-\left(\mathbf{a}^{\prime} \cdot \mathbf{b}^{\prime}\right)^{2}\right)^{-\frac{1}{2}}\left(1+\mathbf{a}^{\prime} \cdot \mathbf{b}^{\prime}, \mathbf{a}^{\prime}+\mathbf{b}^{\prime}\right) \\
& n_{2}^{\mu}=\left(1-\left(\mathbf{a}^{\prime} \cdot \mathbf{b}^{\prime}\right)^{2}\right)^{-\frac{1}{2}}\left(0, \mathbf{a}^{\prime} \wedge \mathbf{b}^{\prime}\right)
\end{aligned}
$$

both of which satisfy the above conditions. Even though $n_{1}^{\mu}$ and $n_{2}^{\mu}$ are not well defined at the cusp, they are well behaved at any distance or time arbitrarily close to it. As we approach the cusp, (which we take to be at $\tau=0, \sigma=0$ as in section 3), $n_{1}^{\mu}$ becomes parallel to $\dot{X}$ and tilts over towards the light cone whereas $n_{2}^{\mu}$ flips direction across the cusp.

The extrinsic curvatures associated with $n_{1}^{\mu}$ and $n_{2}^{\mu}$ are:

$$
K_{i A B}=\frac{\partial X^{\mu}}{\partial \sigma^{A}} \frac{\partial X^{\nu}}{\partial \sigma^{B}} \nabla_{(\mu} n_{i \nu)}=-n_{i \mu} X_{, A B}^{\mu}
$$


Notice that

$$
\begin{gathered}
X^{\prime}=\left(0, \frac{\mathbf{b}^{\prime}-\mathbf{a}^{\prime}}{2}\right), \quad \dot{X}=\left(1, \frac{\mathbf{a}^{\prime}+\mathbf{b}^{\prime}}{2}\right) \\
\Rightarrow \quad X^{\prime \prime}=\ddot{X}=\left(0, \frac{\mathbf{a}^{\prime \prime}+\mathbf{b}^{\prime \prime}}{2}\right), \quad \dot{X}^{\prime}=\left(0, \frac{\mathbf{b}^{\prime \prime}-\mathbf{a}^{\prime \prime}}{2}\right)
\end{gathered}
$$

and hence we can write all second derivatives as:

$$
X_{, A B}^{\mu}=\frac{1}{2}\left(0,\left(\delta_{A}^{\tau} \delta_{B}^{\tau}+\delta_{A}^{\sigma} \delta_{B}^{\sigma}\right)\left(\mathbf{a}^{\prime \prime}+\mathbf{b}^{\prime \prime}\right)+2 \delta_{(A}^{\tau} \delta_{B)}^{\sigma}\left(\mathbf{b}^{\prime \prime}-\mathbf{a}^{\prime \prime}\right)\right) .
$$

Substituting this into (A.5) yields

$$
\begin{aligned}
K_{1 A B}= & \frac{1}{2}\left(1-\left(\mathbf{a}^{\prime} \cdot \mathbf{b}^{\prime}\right)^{2}\right)^{-\frac{1}{2}}\left[\left(\delta_{A}^{\tau} \delta_{B}^{\tau}+\delta_{A}^{\sigma} \delta_{B}^{\sigma}\right)\left(\mathbf{a}^{\prime \prime} \cdot \mathbf{b}^{\prime}+\mathbf{b}^{\prime \prime} \cdot \mathbf{a}^{\prime}\right)+2 \delta_{(A}^{\tau} \delta_{B)}^{\sigma}\left(\mathbf{b}^{\prime \prime} \cdot \mathbf{a}^{\prime}-\mathbf{a}^{\prime \prime} \cdot \mathbf{b}^{\prime}\right)\right] \\
K_{2 A B}= & \frac{1}{2}\left(1-\left(\mathbf{a}^{\prime} \cdot \mathbf{b}^{\prime}\right)^{2}\right)^{-\frac{1}{2}}\left[\left(\delta_{A}^{\tau} \delta_{B}^{\tau}+\delta_{A}^{\sigma} \delta_{B}^{\sigma}\right)\left(\mathbf{a}^{\prime \prime}+\mathbf{b}^{\prime \prime}\right) \cdot\left(\mathbf{a}^{\prime} \wedge \mathbf{b}^{\prime}\right)\right. \\
& \left.+2 \delta_{(A}^{\tau} \delta_{B)}^{\sigma}\left(\mathbf{b}^{\prime \prime}-\mathbf{a}^{\prime \prime}\right) \cdot\left(\mathbf{a}^{\prime} \wedge \mathbf{b}^{\prime}\right)\right]
\end{aligned}
$$

The leading nontrivial finite width correction to the Nambu action appears at fourth order in $w$, the width of the string, and is given by [29, 52]

$$
S=-\mu \int d^{2} \sigma \sqrt{\gamma}\left(1+w^{4}\left[\alpha_{2}\left(\Sigma_{1}+\Sigma_{2}\right)^{2}+2 \alpha_{3}\left(\Sigma_{3}^{2}+\Sigma_{1} \Sigma_{2}\right)\right]\right.
$$

The $\alpha_{n}$ are numerically calculated coefficients dependent on the specific model for the vortex, and for the Nielsen-Olesen vortex were computed in [29] and found to be of order unity. For example, in the supersymmetric Abelian Higgs vortex, $\alpha_{2}=$ $-\alpha_{3} / 2 \sim 3.36$. The $\Sigma_{i}$ are scalars on the worldsheet constructed from the extrinsic curvatures:

$$
\Sigma_{1}=K_{1 A B} K_{1}^{A B}, \quad \Sigma_{2}=K_{2 A B} K_{2}^{A B}, \quad \Sigma_{3}=K_{1 A B} K_{2}^{A B} .
$$

Note that to calculate these scalars we raise the indices of $K_{i A B}$ using the inverse of our worldsheet metric (2.13):

$$
\gamma^{A B}=\frac{2}{\left(1-\mathbf{a}^{\prime} \cdot \mathbf{b}^{\prime}\right)} \eta^{A B}
$$


We thus calculate the worldsheet scalars to be:

$$
\begin{aligned}
\Sigma_{1}= & K_{1 A B} K_{1}^{A B} \\
= & 2\left(1-\left(\mathbf{a}^{\prime} \cdot \mathbf{b}^{\prime}\right)^{2}\right)^{-1}\left(1-\mathbf{a}^{\prime} \cdot \mathbf{b}^{\prime}\right)^{-2}\left[\left(\mathbf{a}^{\prime \prime} \cdot \mathbf{b}^{\prime}+\mathbf{b}^{\prime \prime} \cdot \mathbf{a}^{\prime}\right)^{2}-\left(\mathbf{b}^{\prime \prime} \cdot \mathbf{a}^{\prime}-\mathbf{a}^{\prime \prime} \cdot \mathbf{b}^{\prime}\right)^{2}\right] \\
= & \frac{8\left(\mathbf{a}^{\prime \prime} \cdot \mathbf{b}^{\prime}\right)\left(\mathbf{b}^{\prime \prime} \cdot \mathbf{a}^{\prime}\right)}{\left(1-\left(\mathbf{a}^{\prime} \cdot \mathbf{b}^{\prime}\right)^{2}\right)\left(1-\mathbf{a}^{\prime} \cdot \mathbf{b}^{\prime}\right)^{2}} \\
\Sigma_{2}= & K_{2 A B} K_{2}^{A B} \\
= & 2\left(1-\left(\mathbf{a}^{\prime} \cdot \mathbf{b}^{\prime}\right)^{2}\right)^{-1}\left(1-\mathbf{a}^{\prime} \cdot \mathbf{b}^{\prime}\right)^{-2}\left[\left(\left(\mathbf{a}^{\prime \prime}+\mathbf{b}^{\prime \prime}\right) \cdot\left(\mathbf{a}^{\prime} \wedge \mathbf{b}^{\prime}\right)\right)^{2}-\left(\left(\mathbf{b}^{\prime \prime}-\mathbf{a}^{\prime \prime}\right) \cdot\left(\mathbf{a}^{\prime} \wedge \mathbf{b}^{\prime}\right)\right)^{2}\right] \\
= & \frac{8\left(\mathbf{a}^{\prime \prime} \cdot\left(\mathbf{a}^{\prime} \wedge \mathbf{b}^{\prime}\right)\right)\left(\mathbf{b}^{\prime \prime} \cdot\left(\mathbf{a}^{\prime} \wedge \mathbf{b}^{\prime}\right)\right)}{\left(1-\left(\mathbf{a}^{\prime} \cdot \mathbf{b}^{\prime}\right)^{2}\right)\left(1-\mathbf{a}^{\prime} \cdot \mathbf{b}^{\prime}\right)^{2}} \\
\Sigma_{3}= & K_{1 A B} K_{2}^{A B} \\
= & 2\left(1-\left(\mathbf{a}^{\prime} \cdot \mathbf{b}^{\prime}\right)^{2}\right)^{-1}\left(1-\mathbf{a}^{\prime} \cdot \mathbf{b}^{\prime}\right)^{-2}\left[\left(\mathbf{a}^{\prime \prime} \cdot \mathbf{b}^{\prime}+\mathbf{b}^{\prime \prime} \cdot \mathbf{a}^{\prime}\right)\left(\left(\mathbf{a}^{\prime \prime}+\mathbf{b}^{\prime \prime}\right) \cdot\left(\mathbf{a}^{\prime} \wedge \mathbf{b}^{\prime}\right)\right)\right. \\
& \left.-\left(\mathbf{b}^{\prime \prime} \cdot \mathbf{a}^{\prime}-\mathbf{a}^{\prime \prime} \cdot \mathbf{b}^{\prime}\right)\left(\left(\mathbf{b}^{\prime \prime}-\mathbf{a}^{\prime \prime}\right) \cdot\left(\mathbf{a}^{\prime} \wedge \mathbf{b}^{\prime}\right)\right)\right] \\
= & \frac{4\left[\left(\mathbf{a}^{\prime \prime} \cdot \mathbf{b}^{\prime}\right)\left(\mathbf{b}^{\prime \prime} \cdot\left(\mathbf{a}^{\prime} \wedge \mathbf{b}^{\prime}\right)\right)+\left(\mathbf{b}^{\prime \prime} \cdot \mathbf{a}^{\prime}\right)\left(\mathbf{a}^{\prime \prime} \cdot\left(\mathbf{a}^{\prime} \wedge \mathbf{b}^{\prime}\right)\right)\right]}{\left(1-\left(\mathbf{a}^{\prime} \cdot \mathbf{b}^{\prime}\right)^{2}\right)\left(1-\mathbf{a}^{\prime} \cdot \mathbf{b}^{\prime}\right)^{2}} .
\end{aligned}
$$

In order to determine if these terms will result in a significant correction to the action (A.10), we must examine the scalars' behaviour close to our exact cusp. We perform a Taylor expansion of $\mathbf{a}^{\prime}, \mathbf{b}^{\prime}, \mathbf{a}^{\prime \prime}$ and $\mathbf{b}^{\prime \prime}$ around the cusp position $\sigma_{ \pm}=0$, yielding

$$
\begin{aligned}
& \Sigma_{1} \simeq 32\left[\frac{\left(\mathrm{a}_{0}^{\prime \prime 2} \sigma_{-}-\mathrm{a}_{0}^{\prime \prime} \mathrm{b}_{0}^{\prime \prime} \cos \psi \sigma_{+}\right)\left(\mathrm{b}_{0}^{\prime \prime 2} \sigma_{+}-\mathrm{a}_{0}^{\prime \prime} \mathrm{b}_{0}^{\prime \prime} \cos \psi \sigma_{-}\right)}{\left(a_{0}^{\prime \prime 2} \sigma_{-}^{2}+b_{0}^{\prime \prime 2} \sigma_{+}^{2}-2 a_{0}^{\prime \prime} b_{0}^{\prime \prime} \cos \psi \sigma_{-} \sigma_{+}\right)^{3}}\right] \\
& \Sigma_{2} \simeq 32\left[\frac{\mathrm{a}_{0}^{\prime \prime 2} \mathrm{~b}_{0}^{\prime \prime 2} \sin ^{2} \psi \sigma_{-} \sigma_{+}}{\left(a_{0}^{\prime \prime 2} \sigma_{-}^{2}+b_{0}^{\prime \prime 2} \sigma_{+}^{2}-2 a_{0}^{\prime \prime} b_{0}^{\prime \prime} \cos \psi \sigma_{-} \sigma_{+}\right)^{3}}\right] \\
& \Sigma_{3} \simeq-16\left[\frac{\mathrm{a}_{0}^{\prime \prime} \mathrm{b}_{0}^{\prime \prime} \sin \psi}{\left(a_{0}^{\prime \prime 2} \sigma_{-}^{2}+b_{0}^{\prime \prime 2} \sigma_{+}^{2}-2 a_{0}^{\prime \prime} b_{0}^{\prime \prime} \cos \psi \sigma_{-} \sigma_{+}\right)^{2}}\right],
\end{aligned}
$$

where $\psi$ is the angle between $\mathbf{a}_{\mathbf{0}}^{\prime \prime}$ and $\mathbf{b}_{\mathbf{0}}^{\prime \prime}$ and we use $\mathrm{a}_{0}^{\prime \prime}$ and $\mathrm{b}_{0}^{\prime \prime}$ to refer to the magnitude of their corresponding vectors. We now fix $\sigma=0$ and allow $\tau$ to vary in order to see how the string curvature behaves as the cusp forms:

$$
\Sigma_{1} \simeq 32\left[\frac{\left(\mathrm{a}_{0}^{\prime \prime 2}-\mathrm{a}_{0}^{\prime \prime} \mathrm{b}_{0}^{\prime \prime} \cos \psi\right)\left(\mathrm{b}_{0}^{\prime \prime 2}-\mathrm{a}_{0}^{\prime \prime} \mathrm{b}_{0}^{\prime \prime} \cos \psi\right)}{\left(a_{0}^{\prime \prime 2}+b_{0}^{\prime \prime 2}-2 a_{0}^{\prime \prime} b_{0}^{\prime \prime} \cos \psi\right)^{3}}\right] \tau^{-4}, \quad \sigma=0, \tau \ll L .
$$

Alternatively, we can investigate the shape of the string at the precise moment of the cusp by keeping $\tau=0$ and allowing $\sigma$ to vary:

$$
\Sigma_{1} \simeq-32\left[\frac{\left(\mathrm{a}_{0}^{\prime \prime 2}+\mathrm{a}_{0}^{\prime \prime} \mathrm{b}_{0}^{\prime \prime} \cos \psi\right)\left(\mathrm{b}_{0}^{\prime \prime 2}+\mathrm{a}_{0}^{\prime \prime} \mathrm{b}_{0}^{\prime \prime} \cos \psi\right)}{\left(a_{0}^{\prime \prime 2}+b_{0}^{\prime \prime 2}+2 a_{0}^{\prime \prime} b_{0}^{\prime \prime} \cos \psi\right)^{3}}\right] \sigma^{-4}, \quad \tau=0, \sigma \ll L .
$$


We can see that as the cusp is formed $(\tau \rightarrow 0)$, the extrinsic curvature scalars $\Sigma_{i}$ grow rapidly, diverging as $\sigma_{ \pm}^{-4}$ and becoming infinite at the cusp itself $\left(\Sigma_{2}\right.$ and $\Sigma_{3}$ exhibit similar behaviour to $\Sigma_{1}$ ). These terms can therefore no longer be neglected and it would seem that we must indeed include the leading order correction in (A.10).

However, we note that, as in section 3, we can take the order of magnitude of $a_{0}^{\prime \prime} \sim b_{0}^{\prime \prime} \sim 2 \pi / L$ (see 31 also), implying

$$
\Sigma_{i} \sim \frac{L^{2}}{\sigma_{ \pm}^{4}} .
$$

Thus the corrections to the Nambu action become non-negligible for

$$
\Sigma_{i} \sim \frac{1}{w^{2}}
$$

i.e.

$$
\sigma_{ \pm} \sim(w L)^{1 / 2}
$$

We therefore conclude that the Nambu action (1.1) will not break down and our analysis remains valid provided we take

$$
\sigma_{ \pm} \gg(w L)^{1 / 2}
$$

As we mentioned in section 5, the width of the string $w$ is set by the inflationary scale, while the physical scale of interest is cosmological, and therefore $(w L)^{1 / 2}$ will be extremely small, making it possible to fulfil this condition.

The idea is therefore that if an exact cusp is supposed to occur at $\sigma_{ \pm}=0$, where the $\mathbf{a}^{\prime}$ and $\mathbf{b}^{\prime}$ curves cross, then at some point before the event (e.g. at $\tau \sim(w L)^{1 / 2}$ and $\left.\sigma=0\right)$, the condition $\sigma_{ \pm}>\sigma_{\min } \sim(w L)^{1 / 2}$ is broken and the Nambu approximation breaks down at that moment. It has been argued that as the cusp forms, the two string segments close to the point of the cusp could overlap (c.f. figure 1 of [54]), resulting in a small loop separating from the string (due to it reconnecting), along with the consequent particle emissions. This would then result in a bridging effect and a rerouting of the trajectories on the Kibble-Turok sphere [53, 54]. Given that Olum and Blanco-Pillado also show that the size of the overlapping segment is of the same order as the value of $\sigma_{ \pm}$in (A.23), (i.e. where we believe the curvature effects are becoming relevant), it would seem that using the analytical description found from the Nambu action to calculate the overlap at this point is unjustified. However, the general picture of an additional emission of energy of the same order as the energy in that segment of the string, consistent with the simulation in [54], still seems feasible since we expect that as the curvature starts to diverge, the string must somehow round off to avoid an exact cusp.

We now use a simple method of estimation to check if GWB's from a cusp will be affected by the imposition of a lower bound of $\sigma_{\min } \sim(w L)^{1 / 2}$, allowing us to 
continue using the Nambu approximation. If we consider eqn (3.9) and set $\varepsilon=0$, (i.e. $k^{\mu} \| \ell^{\mu}$ ), the integrals $I_{ \pm}$can be estimated by

$$
I=\int_{-\infty}^{\infty} d u u e^{ \pm i u^{3}}
$$

where $u$ is given by (3.8) as before. However the real limits of this integral should be determined by the limits on $\sigma_{ \pm}$. In other words (A.25) is an approximation of

$$
I_{\sigma}=\int_{-u_{\max }}^{u_{\max }} d u u e^{ \pm i u^{3}}-\int_{-u_{\min }}^{u_{\min }} d u u e^{ \pm i u^{3}}
$$

where $u_{\min } \equiv u\left(\sigma_{\min }\right)$ and $u_{\max } \equiv u(L)$. We illustrate how these limits influence the integral's behaviour in figure 8. We can see from this plot that the approximate

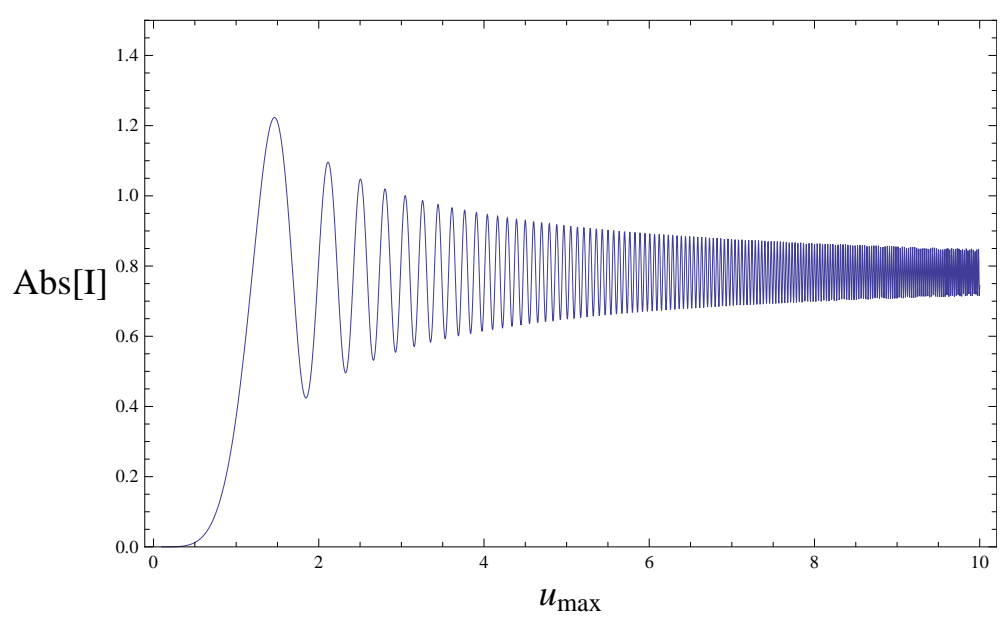

Figure 8: The value of the integral $I$ (A.25) integrated between $\pm u_{\max }$ for various values of $u_{\max }$.

value of 0.7818 obtained from (A.25) is roughly within $20 \%$ of the exact value of $I_{\sigma}$ if we assume that $u_{\min } \lesssim 0.5$ and $u_{\max } \gtrsim 4$. We now proceed to calculate the value of $u_{\text {min }}$ using our lower bound of $\sigma_{\min } \sim(w L)^{1 / 2}$ :

$$
\begin{aligned}
u_{\text {min }} & \sim\left[\frac{1}{12}|m| \omega_{L}\left(\ddot{X}_{ \pm}\right)^{2}\right]^{\frac{1}{3}} \cdot \sigma_{\min } \\
& \sim\left[\frac{1}{12} \cdot 2 \pi f \cdot\left(\frac{x_{2}}{L}\right)^{2}\right]^{\frac{1}{3}} \cdot \sigma_{m i n} \\
& \sim\left[\frac{\pi}{6} f\left(\frac{x_{2}}{L}\right)^{2}\right]^{\frac{1}{3}} \cdot(w L)^{1 / 2}
\end{aligned}
$$

where we have substituted $f=m \omega_{L} / 2 \pi$ as before and $\ddot{X}_{ \pm} \sim x_{2} / L$. We introduce $x_{2}$ as a naive but simple way of incorporating the number of harmonics in the string solution and as an attempt to account for how wiggly the string is. In the DV 
approximation it is taken to be of order one (we have used $\ddot{X}_{ \pm} \sim 2 \pi / L$ previously). We again use the one scale model (3.14) with $\alpha \sim \Gamma G \mu$ and $\Gamma \sim 50$ and the DV interpolating function $t \sim t_{0}(1+z)^{-3 / 2}\left(1+z / z_{e q}\right)^{-1 / 2}$ [31. Now if we use our fiducial frequency $f=150 \mathrm{~Hz} \sim 10^{-22} \mathrm{GeV}$, which is in the optimal frequency range of LIGO, and $10^{-7}>G \mu>10^{-12}, t_{0}^{-1} \sim H_{0} \sim 10^{-42} \mathrm{GeV}, G \sim 10^{-38} \mathrm{GeV}^{-2}$ and $w \sim \frac{1}{\sqrt{\mu}}$, we get:

$$
\begin{aligned}
u_{\text {min }} & \sim f^{1 / 3} x_{2}^{2 / 3}(\Gamma G \mu)^{-1 / 6} t^{1 / 6} \mu^{1 / 4} \\
& \sim 10^{-7} x_{2}^{2 / 3} \Gamma^{-1 / 6}(G \mu)^{-1 / 6} \mu^{1 / 4} H_{0}^{1 / 6}(1+z)^{1 / 4}\left(1+\frac{z}{z_{e q}}\right)^{1 / 12} \\
& \sim 10^{-14} 10^{-9.5} x_{2}^{2 / 3} \Gamma^{-1 / 6}(G \mu)^{-5 / 12}(1+z)^{1 / 4}\left(1+\frac{z}{z_{e q}}\right)^{1 / 12} \\
& \sim 10^{-18} x_{2}^{2 / 3}\left(\frac{f_{H z}}{150}\right)^{1 / 3}\left(\frac{\Gamma}{50}\right)^{-1 / 6}\left(\frac{G \mu}{10^{-12}}\right)^{-5 / 12}(1+z)^{1 / 4}\left(1+\frac{z}{z_{e q}}\right)^{1 / 12}
\end{aligned}
$$

We see that as long as $x_{2}$ is not drastically large (i.e. the string is extremely wiggly) or $\Gamma$ is not significantly smaller than $50, u_{\min } \ll 1$ is a valid approximation for any feasible gravitational wave experiment and none of the other parameters in the above expression can compensate for the smallness of the size of the cusp segment compared to the size of the loop. Indeed, even for very high redshifts up to $z \sim 10^{12}$ corresponding to $100 \mathrm{MeV}$ scales, the corrections at most change the result of $u_{\text {min }}$ by a few orders of magnitude.

We perform a similar calculation to approximate $u_{\max }$ using $\sigma_{\max }=L$ :

$$
\begin{aligned}
u_{\text {max }} & \sim\left[\frac{\pi}{6} f\left(\frac{x_{2}}{L}\right)^{2}\right]^{\frac{1}{3}} \cdot L \\
& \sim 10^{3} x_{2}^{2 / 3}\left(\frac{f_{H z}}{150}\right)^{1 / 3}\left(\frac{\Gamma}{50}\right)^{1 / 3}\left(\frac{G \mu}{10^{-12}}\right)^{1 / 3}(1+z)^{-1 / 2}\left(1+\frac{z}{z_{e q}}\right)^{-1 / 6}
\end{aligned}
$$

which suggests that the approximation remains valid at least up to $z \leq 10^{8}$ or $10^{-2}$ $\mathrm{MeV}$ scales. Hence (A.25) is a valid approximation of (A.26) for our limits on $\sigma_{ \pm}$.

We have therefore shown that the use of the Nambu action (1.1), rather than the corrected action (A.10), in calculating GWB's from an exact cusp is justified.

\section{References}

[1] V. A. Rubakov and M. E. Shaposhnikov, Phys. Lett. B 125, 139 (1983).

V. A. Rubakov and M. E. Shaposhnikov, Phys. Lett. B 125, 136 (1983).

K. Akama, Lect. Notes Phys. 176, 267 (1982). [arXiv:hep-th/0001113].

[2] N. Arkani-Hamed, S. Dimopoulos and G. Dvali, Phys. Lett. B429, 263 (1998)

[hep-ph/9803315]. 
N. Arkani-Hamed, S. Dimopoulos and G. Dvali, Phys. Rev. D 59, 086004 (1999) [hep-ph/9807344].

I. Antoniadis, N. Arkani-Hamed, S. Dimopoulos and G. Dvali, Phys. Lett. B 436, 257 (1998) [hep-ph/9804398].

[3] L. Randall and R. Sundrum, Phys. Rev. Lett. 83, 3370 (1999) [arXiv:hep-ph/9905221]. L. Randall and R. Sundrum, Phys. Rev. Lett. 83, 4690 (1999) [arXiv:hep-th/9906064].

[4] S. B. Giddings, S. Kachru and J. Polchinski, Phys. Rev. D 66, 106006 (2002).

S. Kachru, R. Kallosh, A. Linde, and S. P. Trivedi, Phys. Rev. D 68, 046005 (2003).

[5] H. A. Chamblin and H. S. Reall, Nucl. Phys. B 562, 133 (1999) [arXiv:hep-th/9903225].

N. Kaloper, Phys. Rev. D 60, 123506 (1999) [arXiv:hep-th/9905210].

P. Kraus, JHEP 9912, 011 (1999) [arXiv:hep-th/9910149].

P. Bowcock, C. Charmousis and R. Gregory, Class. Quant. Grav. 17, 4745 (2000) [arXiv:hep-th/0007177].

[6] A. Kehagias and E. Kiritsis, JHEP 9911, 022 (1999) [arXiv:hep-th/9910174].

[7] C. Charmousis, R. Emparan and R. Gregory, JHEP 0105, 026 (2001) [arXiv:hep-th/0101198].

J. M. Cline, J. Descheneau, M. Giovannini and J. Vinet, JHEP 0306, 048 (2003) [arXiv:hep-th/0304147].

C. Charmousis and R. Gregory, Class. Quant. Grav. 21, 527 (2004) [arXiv:gr-qc/0306069].

[8] G. R. Dvali and S. H. H. Tye, Phys. Lett. B 450, 72 (1999) [arXiv:hep-ph/9812483].

S. H. S. Alexander, Phys. Rev. D 65, 023507 (2002) [arXiv:hep-th/0105032].

C. P. Burgess, M. Majumdar, D. Nolte, F. Quevedo, G. Rajesh and R. J. Zhang, JHEP 0107, 047 (2001) [arXiv:hep-th/0105204].

G. Shiu and S. H. H. Tye, Phys. Lett. B 516, 421 (2001) [arXiv:hep-th/0106274];

[9] S. Kachru, R. Kallosh, A. D. Linde, J. M. Maldacena, L. P. McAllister and S. P. Trivedi, JCAP 0310, 013 (2003) [arXiv:hep-th/0308055].

[10] R. Kallosh, Lect. Notes Phys. 738, 119 (2008) [arXiv:hep-th/0702059]. L. McAllister and E. Silverstein, Gen. Rel. Grav. 40, 565 (2008) [arXiv:0710.2951 [hep-th]].

[11] S. Sarangi and S. H. H. Tye, Phys. Lett. B 536, 185 (2002) [arXiv:hep-th/0204074]. G. Dvali and A. Vilenkin, Phys. Rev. D 67, 046002 (2003) [arXiv:hep-th/0209217]. G. Dvali and A. Vilenkin, JCAP 0403, 010 (2004) [arXiv:hep-th/0312007].

[12] N. T. Jones, H. Stoica and S. H. H. Tye, Phys. Lett. B 563 (2003) 6 [arXiv:hep-th/0303269]. 
E. J. Copeland, R. C. Myers and J. Polchinski, JHEP 0406, 013 (2004) [arXiv:hep-th/0312067].

[13] J. Polchinski, "Introduction to cosmic F- and D-strings," arXiv:hep-th/0412244. E. J. Copeland and T. W. B. Kibble, "Cosmic Strings and Superstrings," arXiv:0911.1345 [Unknown].

[14] A. Vilenkin, Phys. Rept. 121, 263 (1985).

M. B. Hindmarsh and T. W. B. Kibble, Rept. Prog. Phys. 58, 477 (1995) [arXiv:hep-ph/9411342].

[15] Y. B. Zeldovich, I. Y. Kobzarev and L. B. Okun, Zh. Eksp. Teor. Fiz. 67, 3 (1974) [Sov. Phys. JETP 40, 1 (1974)].

T. W. B. Kibble, J. Phys. A 9, 1387 (1976).

[16] B. Allen and E. P. S. Shellard, Phys. Rev. Lett. 64, 119 (1990).

D. P. Bennett and F. R. Bouchet, Phys. Rev. D 41, 2408 (1990).

[17] A. J. Albrecht, R. A. Battye and J. Robinson, Phys. Rev. Lett. 79, 4736 (1997) [arXiv:astro-ph/9707129].

[18] M. Wyman, L. Pogosian and I. Wasserman, Phys. Rev. D 72, 023513 (2005)

[Erratum-ibid. D 73, 089905 (2006)] [arXiv:astro-ph/0503364].

N. Bevis, M. Hindmarsh, M. Kunz and J. Urrestilla, Phys. Rev. Lett. 100, 021301 (2008) [arXiv:astro-ph/0702223].

[19] A. Vilenkin, Phys. Rev. D 23, 852 (1981).

J. R. I. Gott, Astrophys. J. 288, 422 (1985).

W. A. Hiscock, Phys. Rev. D 31, 3288 (1985).

B. Linet, Gen. Rel. Grav. 17, 1109 (1985).

D. Garfinkle, Phys. Rev. D 32, 1323 (1985).

R. Gregory, Phys. Rev. Lett. 59, 740 (1987).

[20] K. Kuijken, X. Siemens and T. Vachaspati, Mon. Not. R. Astron. Soc. 384, 161-164 (2008). [arXiv:0707.2971 [astro-ph]].

D. F. Chernoff and S. H. H. Tye, "Cosmic String Detection via Microlensing of Stars," arXiv:0709.1139 [astro-ph].

[21] T. Vachaspati, A. E. Everett and A. Vilenkin, Phys. Rev. D 30, 2046 (1984).

T. Vachaspati and A. Vilenkin, Phys. Rev. D 31, 3052 (1985).

C. J. Burden, Phys. Lett. B 164, 277 (1985).

T. Vachaspati, Phys. Rev. D 35 (1987) 1767.

[22] D. Garfinkle and T. Vachaspati, Phys. Rev. D 36, 2229 (1987).

F. S. Accetta and L. M. Krauss, Nucl. Phys. B 319, 747 (1989).

R. J. Scherrer, J. M. Quashnock, D. N. Spergel and W. H. Press, Phys. Rev. D 42, 1908 (1990).

M. Sakellariadou, Phys. Rev. D 42, 354 (1990) [Erratum-ibid. D 43, 4150 (1991)]. 
[23] J. M. Quashnock and D. N. Spergel, Phys. Rev. D 42, 2505 (1990).

B. Allen and E. P. S. Shellard, Phys. Rev. D 45, 1898 (1992).

B. Allen, P. Casper and A. Ottewill, Phys. Rev. D 50, 3703 (1994) [arXiv:gr-qc/9405037].

[24] D. Forster, Nucl. Phys. B 81, 84 (1974).

[25] E. P. S. Shellard, Nucl. Phys. B 283, 624 (1987).

K. J. M. Moriarty, E. Myers and C. Rebbi, Phys. Lett. B 207, 411 (1988).

R. A. Matzner, Comput. Phys. 2, 51 (1989).

[26] T. W. B. Kibble and N. Turok, Phys. Lett. B 116, 141 (1982).

N. Turok, Nucl. Phys. B 242, 520 (1984).

[27] D. Delaney, K. Engle and X. Scheick, Phys. Rev. D 41, 1775 (1990).

R. W. Brown, M. E. Convery, S. A. Hotes, M. G. Knepley and L. S. Petropoulos, Phys. Rev. D 48, 2548 (1993) [arXiv:hep-th/9307077].

B. Allen and P. Casper, Phys. Rev. D 50, 2496 (1994) [arXiv:gr-qc/9405005].

[28] R. Gregory, Phys. Lett. B 206, 199 (1988).

R. Gregory, D. Haws and D. Garfinkle, Phys. Rev. D 42, 343 (1990).

[29] M. R. Anderson, F. Bonjour, R. Gregory and J. Stewart, Phys. Rev. D 56 (1997) 8014 [arXiv:hep-ph/9707324].

[30] X. Siemens and K. D. Olum, Nucl. Phys. B 611, 125 (2001) [Erratum-ibid. B 645, 367 (2002)] [arXiv:gr-qc/0104085].

[31] T. Damour and A. Vilenkin, Phys. Rev. D 64 (2001) 064008 [arXiv:gr-qc/0104026].

[32] T. Damour and A. Vilenkin, Phys. Rev. D 71 (2005) 063510 [arXiv:hep-th/0410222].

[33] X. Siemens, J. Creighton, I. Maor, S. Ray Majumder, K. Cannon and J. Read, Phys. Rev. D 73, 105001 (2006) [arXiv:gr-qc/0603115].

[34] X. Siemens, V. Mandic and J. Creighton, Phys. Rev. Lett. 98, 111101 (2007) [arXiv:astro-ph/0610920].

[35] A. C. Davis, W. Nelson, S. Rajamanoharan and M. Sakellariadou, JCAP 0811, 022 (2008) [arXiv:0809.2263 [hep-th]].

R. Brandenberger, H. Firouzjahi, J. Karouby and S. Khosravi, JCAP 0901, 008 (2009) [arXiv:0810.4521 [hep-th]].

P. Binetruy, A. Bohe, T. Hertog and D. A. Steer, Phys. Rev. D 80, 123510 (2009) [arXiv:0907.4522 [hep-th]].

[36] L. Leblond, B. Shlaer and X. Siemens, Phys. Rev. D 79, 123519 (2009) [arXiv:0903.4686 [astro-ph.CO]]. 
[37] E. O'Callaghan, S. Chadburn, G. Geshnizjani, R. Gregory and I. Zavala, "On detection of extra dimensions with gravitational waves from cosmic strings," arXiv:1003.4395 [Unknown].

[38] A. Avgoustidis and E. P. S. Shellard, Phys. Rev. D 71, 123513 (2005) [arXiv:hep-ph/0410349].

[39] J. Polchinski, Phys. Lett. B 209, 252 (1988).

M. G. Jackson, N. T. Jones and J. Polchinski, JHEP 0510, 013 (2005) [arXiv:hep-th/0405229].

A. Hanany and K. Hashimoto, JHEP 0506, 021 (2005) [arXiv:hep-th/0501031].

[40] A. Avgoustidis and E. P. S. Shellard, Phys. Rev. D 73, 041301 (2006) [arXiv:astro-ph/0512582].

[41] F. A. Jenet et al., Astrophys. J. 653, 1571 (2006) [arXiv:astro-ph/0609013].

B. P. Abbott et al. [LIGO Scientific Collaboration], Phys. Rev. D 80, 062002 (2009) [arXiv:0904.4718 [astro-ph.CO]].

[42] X. Siemens, K. D. Olum and A. Vilenkin, Phys. Rev. D 66, 043501 (2002) [arXiv:gr-qc/0203006].

J. Polchinski and J. V. Rocha, Phys. Rev. D 75, 123503 (2007) [arXiv:gr-qc/0702055].

J. V. Rocha, Phys. Rev. Lett. 100, 071601 (2008) [arXiv:0709.3284 [gr-qc]].

[43] D. B. DeLaney and R. W. Brown, Phys. Rev. Lett. 63, 474 (1989).

[44] A. Avgoustidis, Phys. Rev. D 78, 023501 (2008) [arXiv:0712.3224 [hep-th]].

[45] E. Witten, Nucl. Phys. B 249, 557 (1985).

[46] N. K. Nielsen, Nucl. Phys. B 167, 249 (1980). N. K. Nielsen and P. Olesen, Nucl. Phys. B 291, 829 (1987). J. J. Blanco-Pillado, K. D. Olum and A. Vilenkin, Phys. Rev. D 63, 103513 (2001) [arXiv:astro-ph/0004410].

[47] J. J. Blanco-Pillado and K. D. Olum, Nucl. Phys. B 599, 435 (2001) [arXiv:astro-ph/0008297].

[48] I. Moss and S. J. Poletti, Phys. Lett. B 199, 34 (1987).

[49] M. N. Butler, R. A. Malaney and M. B. Mijic, Phys. Rev. D 43, 2535 (1991).

K. Dimopoulos and A. C. Davis, Phys. Lett. B 446, 238 (1999) [arXiv:hep-ph/9901250].

[50] P. Laguna and R. A. Matzner, Phys. Rev. D 41, 1751 (1990).

[51] A. L. Chen, D. A. DiCarlo and S. A. Hotes, Phys. Rev. D 37, 863 (1988).

[52] M. Anderson, Phys. Rev. D 51, 2863 (1995). 
[53] J. J. Blanco-Pillado and K. D. Olum, Phys. Rev. D 59 (1999) 063508 [arXiv:gr-qc/9810005].

[54] K. D. Olum and J. J. Blanco-Pillado, Phys. Rev. D 60 (1999) 023503 [arXiv:gr-qc/9812040]. 